TRANSACTIONS OF THE

AMERICAN MATHEMATICAL SOCIETY

Volume 355 , Number 4 , Pages 1537-1557

S 0002-9947(02)03184-7

Article electronically published on December 4, 2002

\title{
ON EXTENDABILITY OF GROUP ACTIONS ON COMPACT RIEMANN SURFACES
}

\author{
EMILIO BUJALANCE, F. J. CIRRE, AND MARSTON CONDER
}

\begin{abstract}
The question of whether a given group $G$ which acts faithfully on a compact Riemann surface $X$ of genus $g \geq 2$ is the full group of automorphisms of $X$ (or some other such surface of the same genus) is considered. Conditions are derived for the extendability of the action of the group $G$ in terms of a concrete partial presentation for $G$ associated with the relevant branching data, using Singerman's list of signatures of Fuchsian groups that are not finitely maximal. By way of illustration, the results are applied to the special case where $G$ is a non-cyclic abelian group.
\end{abstract}

\section{INTRODUCTION}

An abstract finite group $G$ is said to act on genus $g \geq 2$ if it is (isomorphic to) a group of automorphisms of some compact Riemann surface of genus $g$. We say that $G$ acts as a full group on genus $g$ if $G$ is the full automorphism group of some compact Riemann surface of genus $g$. An interesting problem in Riemann surface theory is to investigate whether a group $G$ acting on genus $g$ also acts as a full group on genus $g$.

In this paper we study this question of extendability in terms of the branching data of the quotient surface under the action of $G$. The first results in this subject were obtained by Greenberg in [6], who showed, among other things, that every finite group acts as the full automorphism group of some Riemann surface; see also [7. The most fruitful way to approach this problem is through uniformization of Riemann surfaces by means of Fuchsian groups and the theory of Teichmüller spaces.

A compact Riemann surface $X$ of genus $g \geq 2$ can be represented as the quotient $U / \Lambda$ of the hyperbolic plane $U$ under the action of a surface Fuchsian group $\Lambda$. Here, by a Fuchsian group we mean a discrete cocompact subgroup of $\operatorname{PSL}(2, \mathbb{R})$. A group $G$ then acts as a group of automorphisms of $X$ if and only if $G$ is isomorphic to the quotient $\Gamma / \Lambda$ for some Fuchsian group $\Gamma$ containing $\Lambda$ as a normal subgroup of index $|G|$. In this case, $G=$ Aut $X$ (the full automorphism group of $X$ ) if and only if $\Gamma$ is the normaliser in $\operatorname{Aut} U=\operatorname{PSL}(2, \mathbb{R})$ of the surface group $\Lambda$, and hence $G$ fails to be the full automorphism group of $X$ if and only if $\Gamma$ is properly contained

Received by the editors December 10, 2001.

2000 Mathematics Subject Classification. Primary 20H10; Secondary 14H55, 20F38, 30F10.

Key words and phrases. Riemann surface, automorphism group.

The first author was partially supported by DGICYT PB98-0017.

The second author was partially supported by DGICYT PB98-0756.

The third author was partially supported by N.Z. Marsden Fund UOA-810. 
with finite index in another Fuchsian group $\Gamma^{\prime}$ which also normalises $\Lambda$. Accordingly, the above problem is closely related to the finite-index extendability of Fuchsian groups.

The extendability of $\Gamma$ depends mainly on the geometry of a fundamental region for $\Gamma$. This implies that although $\Gamma$ may be contained in a Fuchsian group $\Gamma^{\prime}$ normalising $\Lambda$ (in which case $G \neq \operatorname{Aut} X$ ), the group $\Gamma$ may be abstractly isomorphic to another Fuchsian group $\Gamma_{1}$ which is not contained with finite index in any other Fuchsian group (in which case $G$ does act as a full group on the appropriate genus $g)$. Let $R(\Gamma)$ be the set of all monomorphisms $\rho: \Gamma \rightarrow \operatorname{PSL}(2, \mathbb{R})$ such that $\rho(\Gamma)$ is also a Fuchsian group. Note that every such $\rho$ maps hyperbolic (respectively elliptic) to hyperbolic (resp. elliptic) elements. Greenberg [6] showed that for some $\Gamma$ there exists a Fuchsian group $\Gamma^{\prime}$ containing $\Gamma$ with finite index such that each $\rho \in R(\Gamma)$ extends to some $\rho^{\prime} \in R\left(\Gamma^{\prime}\right)$.

Singerman studied this question in detail in [10], giving a complete list of such groups. The associated signatures appear in Table 1 below, the first eight corresponding to normal extensions and the last eleven corresponding to cases where $\Gamma$ is not normal in $\Gamma^{\prime}$. Singerman's list plays a key role in this paper. Indeed, if $\Gamma$ is a Fuchsian group whose signature does not appear in the first column of Table 1, then $\Gamma$ is isomorphic to a finitely-maximal Fuchsian group, that is, a group that is not contained with finite index in any other Fuchsian group. Accordingly, if the group $G$ can be written as $\Gamma / \Lambda$, where the signature of $\Gamma$ does not appear in Singerman's table, then $G$ acts as a full group on the corresponding genus $g$.

TABLE 1. Non-maximal Fuchsian signatures

\begin{tabular}{|l|l|l|c|}
\hline Case & Signature $\sigma=\sigma(\Gamma)$ & \multicolumn{1}{|c|}{$\sigma^{\prime}=\sigma\left(\Gamma^{\prime}\right)$} & $\left|\Gamma^{\prime}: \Gamma\right|$ \\
\hline N1 & $(2 ;-)$ & $(0 ; 2,2,2,2,2,2)$ & 2 \\
N2 & $(1 ; t, t)$ & $(0 ; 2,2,2,2, t)$ & 2 \\
N3 & $(1 ; t)$ & $(0 ; 2,2,2,2 t)$ & 2 \\
N4 & $(0 ; t, t, t, t), t \geq 3$ & $(0 ; 2,2,2, t)$ & 4 \\
N5 & $(0 ; t, t, u, u), t+u \geq 5$ & $(0 ; 2,2, t, u)$ & 2 \\
N6 & $(0 ; t, t, t), t \geq 4$ & $(0 ; 3,3, t)$ & 3 \\
N7 & $(0 ; t, t, t), t \geq 4$ & $(0 ; 2,3,2 t)$ & 6 \\
N8 & $(0 ; t, t, u), t \geq 3, t+u \geq 7$ & $(0 ; 2, t, 2 u)$ & 2 \\
\hline T1 & $(0 ; 7,7,7)$ & $(0 ; 2,3,7)$ & 24 \\
T2 & $(0 ; 2,7,7)$ & $(0 ; 2,3,7)$ & 9 \\
T3 & $(0 ; 3,3,7)$ & $(0 ; 2,3,7)$ & 8 \\
T4 & $(0 ; 4,8,8)$ & $(0 ; 2,3,8)$ & 12 \\
T5 & $(0 ; 3,8,8)$ & $(0 ; 2,3,8)$ & 10 \\
T6 & $(0 ; 9,9,9)$ & $(0 ; 2,3,9)$ & 12 \\
T7 & $(0 ; 4,4,5)$ & $(0 ; 2,4,5)$ & 6 \\
T8 & $(0 ; n, 4 n, 4 n), n \geq 2$ & $(0 ; 2,3,4 n)$ & 6 \\
T9 & $(0 ; n, 2 n, 2 n), n \geq 3$ & $(0 ; 2,4,2 n)$ & 4 \\
T10 & $(0 ; 3, n, 3 n), n \geq 3$ & $(0 ; 2,3,3 n)$ & 4 \\
T11 & $(0 ; 2, n, 2 n), n \geq 4$ & $(0 ; 2,3,2 n)$ & 3 \\
\hline
\end{tabular}


The study of maximality of actions of groups on Riemann surfaces may proceed in two directions:

One of them is the following: fix a family of Riemann surfaces and then find all groups which act as a full group of automorphisms of some surface in the given family. For example, the case of surfaces of low genus was carefully studied by Wiman [14 in the late 1800s. Wiman directly handled defining equations of the surfaces, as used also in [5], [12] and [13. Similarly, the technique of Fuchsian groups was used in [4 to solve the problem for the family of hyperelliptic Riemann surfaces.

The other direction is the opposite of the first: fix a class of finite groups and then study the Riemann surfaces on which a group in the given class acts as a full group of automorphisms. The class of cyclic groups is of course the most extensively studied. In [8], Harvey determined the signatures of those Fuchsian groups $\Gamma$ for which $\Gamma / \Lambda$ is cyclic, where $\Lambda$ is a surface group, and the extendability of such groups was considered in [3] and also in [11. Non-cyclic abelian groups were investigated in [9], where Maclachlan found the minimum genus of surfaces admitting a group of this type as an automorphism group.

In this paper we adopt a more intrinsic point of view for the problem of extendability of groups, restricting neither to a particular class of groups nor to a fixed family of surfaces. We find that in most cases the extendability of the action of a group $G$ depends on the existence of specific types of group automorphisms of $G$, each depending on a concrete partial group presentation of $G$.

Following some further background material in Section 2 we analyse each of the cases from Singerman's list in Sections 3 (normal extensions) and 4 (non-normal extensions) in turn, and then derive extendability theorems in Section 5. Finally, we illustrate the results of our investigation by giving some examples in Section 6 and dealing with the special case of non-cyclic abelian groups in Section 7.

\section{Preliminaries}

The following definitions help us to state the results of the paper.

Definition 2.1. We say that the finite group $G$ acts on genus $g$ if $G$ is (isomorphic to) a group of automorphisms of some compact Riemann surface $X$ of genus $g$. Further, we say that $G$ acts as a full group on genus $g$ if $G$ is the full automorphism group of some compact Riemann surface of genus $g$.

Suppose $G$ acts on genus $g$, and let $X$ be a compact Riemann surface for which $G \subseteq$ Aut $X$. Write $G=\Gamma / \Lambda$, where $\Gamma$ and $\Lambda$ are Fuchsian groups such that $\Lambda$ has signature $(g ;-)$ and is normal in $\Gamma$. If $\Gamma$ has signature $\left(\gamma ; m_{1}, \ldots, m_{r}\right)$, then we say that $G$ acts on genus $g$ with signature $\left(\gamma ; m_{1}, \ldots, m_{r}\right)$, and further, if $G=$ Aut $X$, then we say that $G$ acts as a full group on genus $g$ with signature $\left(\gamma ; m_{1}, \ldots, m_{r}\right)$. Of course $G$ may act with different signatures on the same genus $g$.

Note that in the case of signature $\left(\gamma ; m_{1}, \ldots, m_{r}\right)$, the quotient surface $X / G$ has the structure of a hyperbolic 2-orbifold with $r$ conic points of orders $m_{1}, \ldots, m_{r}$ and underlying topological space of genus $\gamma$. The integers involved must satisfy the Riemann-Hurwitz formula:

$$
2-2 g=|G|\left(2-2 \gamma-\sum_{i=1}^{r}\left(1-\frac{1}{m_{i}}\right)\right) .
$$


A Fuchsian group $\Gamma$ with signature $\left(\gamma ; m_{1}, \ldots, m_{r}\right)$ has an abstract group presentation in terms of $2 \gamma$ hyperbolic generators $a_{1}, b_{1}, \ldots, a_{\gamma}, b_{\gamma}$ and $r$ elliptic generators $x_{1}, \ldots, x_{r}$ subject to the defining relations

$$
x_{1}^{m_{1}}=\cdots=x_{r}^{m_{r}}=x_{1} \cdots x_{r}\left[a_{1}, b_{1}\right] \cdots\left[a_{\gamma}, b_{\gamma}\right]=1 .
$$

These are called canonical generators. The commutator notation is given by $[a, b]=$ $a b a^{-1} b^{-1}$.

We let $R(\Gamma)$ denote the set of monomorphisms $\rho: \Gamma \rightarrow \operatorname{PSL}(2, \mathbb{R})$ such that $\rho(\Gamma)$ is Fuchsian. Two elements $\rho_{1}, \rho_{2} \in R(\Gamma)$ are said to be equivalent if there exists an angle-preserving homeomorphism $h$ of the upper half plane such that $\rho_{1}(\xi)=h \rho_{2}(\xi) h^{-1}$ for each $\xi \in \Gamma$. The quotient space $T(\Gamma)$ of equivalence classes of monomorphisms is called the Teichmüller space of $\Gamma$; this is a cell of complex dimension $\operatorname{dim}(\Gamma)=3 \gamma-3+r$.

Fuchsian groups with signature $\left(0 ; m_{1}, m_{2}, m_{3}\right)$ are known as triangle groups. The Teichmüller dimension of such groups is $3 \gamma-3+r=0-3+3=0$, and from this fact it follows that all embeddings of an abstract triangle group into $\operatorname{PSL}(2, \mathbb{R})$ are mutually conjugate (see [1, Section 10.6]).

An epimorphism $\theta: \Gamma \rightarrow G$ from $\Gamma$ onto a finite group $G$ is said to be smooth if its kernel is a surface Fuchsian group, that is, if $\operatorname{ker} \theta$ contains no element of finite order. Observe that in this case the images of the canonical generators of $\Gamma$ satisfy the same defining relations as above (among others which make $G$ finite). A presentation of $G$ induced in this way by canonical generators of $\Gamma$ will be called a (partial) monodromy presentation.

\section{Normal EXTEnsions}

As noted in the introduction, if the group $G$ can be written as $\Gamma / \Lambda$, where the signature of $\Gamma$ does not appear in Singerman's table (Table 1), then $G$ acts as a full group on the corresponding genus $g$. Hence we focus our attention on Fuchsian groups $\Gamma$ whose signature $\sigma(\Gamma)$ appears in Singerman's table.

In this section we deal with the cases in which $\Gamma$ admits a normal extension to some $\Gamma^{\prime}$. We describe in detail the case of the first row of Table 1 and the others more briefly. In each case we will consider the presentation for $\Gamma$ with signature $\left(\gamma ; m_{1}, \ldots, m_{r}\right)$ as given in the introduction, namely in terms of $2 \gamma$ hyperbolic generators $a_{1}, b_{1}, \ldots, a_{\gamma}, b_{\gamma}$ and $r$ elliptic generators $x_{1}, \ldots, x_{r}$ satisfying the appropriate relations. Similarly for $\Gamma^{\prime}$, which we observe always has signature of the form $\left(0 ; n_{1}, \ldots, n_{s}\right)$, we use a presentation in terms of $s$ elliptic generators $y_{1}, \ldots, y_{s}$ (but no hyperbolic generators) subject to the defining relations $y_{1}^{n_{1}}=\cdots=y_{s}^{n_{s}}=y_{1} \cdots y_{s}=1$.

Case N1: $\quad \sigma(\Gamma)=(2 ;-) ; \quad \sigma\left(\Gamma^{\prime}\right)=(0 ; 2,2,2,2,2,2)$; index $\left|\Gamma^{\prime}: \Gamma\right|=2$.

In this case the group $\Gamma$ has presentation $\left\langle a_{1}, b_{1}, a_{2}, b_{2} \mid\left[a_{1}, b_{1}\right]\left[a_{2}, b_{2}\right]=1\right\rangle$, while $\Gamma^{\prime}$ has presentation $\left\langle y_{1}, y_{2}, y_{3}, y_{4}, y_{5}, y_{6}\right| y_{1}^{2}=y_{2}^{2}=y_{3}^{2}=y_{4}^{2}=y_{5}^{2}=y_{6}^{2}=$ $\left.y_{1} y_{2} y_{3} y_{4} y_{5} y_{6}=1\right\rangle$. Also, as observed in [3], an embedding of $\Gamma$ as a subgroup of index 2 in $\Gamma^{\prime}$ is given by $a_{1} \mapsto y_{1} y_{2}, b_{1} \mapsto y_{3} y_{2}, a_{2} \mapsto y_{4} y_{5}$ and $b_{2} \mapsto y_{6} y_{5}$, since $\left[y_{1} y_{2}, y_{3} y_{2}\right]=y_{1} y_{2} y_{3} y_{2}\left(y_{1} y_{2}\right)^{-1}\left(y_{3} y_{2}\right)^{-1}=\left(y_{1} y_{2} y_{3}\right)^{2}=\left(y_{6} y_{5} y_{4}\right)^{2}=\left[y_{4} y_{5}, y_{6} y_{5}\right]^{-1}$.

Letting $a, b, c$ and $d$ be the images of the canonical generators $a_{1}, b_{1}, a_{2}$ and $b_{2}$ under the smooth epimorphism $\theta: \Gamma \rightarrow G$, we see that $G$ has the partial monodromy presentation $G=\langle a, b, c, d \mid[a, b][c, d]=\cdots=1\rangle$. 
Now suppose the epimorphism $\theta$ can be extended to another smooth epimorphism $\theta^{\prime}$ from $\Gamma^{\prime}$ onto some group $G^{\prime}$ containing $G$ as a (normal) subgroup of index 2. Notice that under these conditions, $\operatorname{ker} \theta^{\prime}=\operatorname{ker} \theta=\Lambda$, and so $G^{\prime}$ is a group of automorphisms of $X$ larger than $G$.

Let $\alpha$ be the image of $y_{1}$ under $\theta^{\prime}$. Then from the embedding of $\Gamma$ in $\Gamma^{\prime}$ it is easy to see that the images under $\theta^{\prime}$ of the generators of $\Gamma^{\prime}$ are as follows:

$$
\begin{array}{lll}
\theta^{\prime}\left(y_{1}\right)=\alpha, & \theta^{\prime}\left(y_{2}\right)=\alpha a, & \theta^{\prime}\left(y_{3}\right)=a^{-1} \alpha b^{-1}, \\
\theta^{\prime}\left(y_{4}\right)=b \alpha d, & \theta^{\prime}\left(y_{5}\right)=d^{-1} \alpha b^{-1} c, & \theta^{\prime}\left(y_{6}\right)=c^{-1} b \alpha .
\end{array}
$$

Since $\theta^{\prime}$ preserves finite orders, each of these elements of $G^{\prime}$ is an involution (in $\left.G^{\prime} \backslash G\right)$, and of course their product $\theta^{\prime}\left(y_{1}\right) \theta^{\prime}\left(y_{2}\right) \theta^{\prime}\left(y_{3}\right) \theta^{\prime}\left(y_{4}\right) \theta^{\prime}\left(y_{5}\right) \theta^{\prime}\left(y_{6}\right)$ is the identity. Further, conjugation by the element $\alpha \in G^{\prime} \backslash G$ has the following effect on the generators of $G$ :

$$
\begin{aligned}
\alpha: & a \mapsto a^{-1}, & c \mapsto\left(b^{-1} c d\right) c^{-1}\left(b^{-1} c d\right)^{-1}, \\
& b \mapsto a b^{-1} a^{-1}, & d \mapsto\left(b^{-1} c\right) d^{-1}\left(b^{-1} c\right)^{-1} .
\end{aligned}
$$

It follows that if such an extension exists from $\Gamma$ to $\Gamma^{\prime}$ (normalising $\Lambda$ ), then $G$ admits a group automorphism $\alpha$ whose effect on the generators $a, b, c$ and $d$ is given by the above equations.

Conversely, if $G$ is a finite group acting on genus $g$ with signature $(2 ;-)$ such that $G$ admits the above automorphism $\alpha$, then $G$ does not act as a full group on genus $g$. In fact, we claim that the semidirect product $G^{\prime}=G \rtimes_{\alpha} C_{2}$ (where the $C_{2}$ is generated by the automorphism $\alpha$ acting on $G$ as above) is a larger automorphism group of the same surface on which $G$ acts. Since $G=\Gamma / \Lambda$ acts with signature $\sigma(\Gamma)=(2,-)$, there exists a Fuchsian group $\Gamma^{\prime}$ with signature $\sigma\left(\Gamma^{\prime}\right)=(0 ; 2,2,2,2,2,2)$ containing $\Gamma$ as a normal subgroup of index 2 . Since each monomorphism $\rho: \Gamma \rightarrow \operatorname{PSL}(2, \mathbb{R})$ extends to some monomorphism $\rho^{\prime}: \Gamma^{\prime} \rightarrow$ $\operatorname{PSL}(2, \mathbb{R})$, it follows that there exists a Fuchsian group isomorphic to $\Gamma^{\prime}$ in which $\Gamma$ is embedded, as in the beginning of this case. Now for such a group, which we still denote by $\Gamma^{\prime}$, the smooth epimorphism $\theta: \Gamma \rightarrow G$ extends to a smooth epimorphism $\theta^{\prime}: \Gamma^{\prime} \rightarrow G^{\prime}$ given by $\theta^{\prime}\left(y_{1}\right)=\alpha, \theta^{\prime}\left(y_{2}\right)=\alpha a, \theta^{\prime}\left(y_{3}\right)=a^{-1} \alpha b^{-1}, \theta^{\prime}\left(y_{4}\right)=b \alpha d$, $\theta^{\prime}\left(y_{5}\right)=d^{-1} \alpha b^{-1} c$ and $\theta^{\prime}\left(y_{6}\right)=c^{-1} b \alpha$, with kernel $\operatorname{ker} \theta^{\prime}=\operatorname{ker} \theta=\Lambda$. Thus $G^{\prime} \cong \Gamma^{\prime} / \Lambda$ is a group of automorphisms of $X=U / \Lambda$ larger than $G$, and so $G$ is not the full automorphism group of $X$.

Case N2: $\sigma(\Gamma)=(1 ; t, t) ; \quad \sigma\left(\Gamma^{\prime}\right)=(0 ; 2,2,2,2, t) ; \quad\left|\Gamma^{\prime}: \Gamma\right|=2$.

This case is similar to the case N1 above. An embedding of $\Gamma$ in $\Gamma^{\prime}$ may be given by $a_{1} \mapsto y_{3} y_{4}, b_{1} \mapsto y_{5} y_{2} y_{3}, x_{1} \mapsto y_{5}$ and $x_{2} \mapsto y_{2} y_{5} y_{2}$, noting that

$$
\begin{aligned}
y_{5}\left(y_{2} y_{5} y_{2}\right)\left[y_{3} y_{4}, y_{5} y_{2} y_{3}\right] & =y_{5} y_{2} y_{5}\left(y_{2} y_{3} y_{4} y_{5}\right) y_{2} y_{3} y_{4} y_{2} y_{5}^{-1} \\
& =y_{5} y_{2}\left(y_{5} y_{1} y_{2} y_{3} y_{4}\right) y_{2} y_{5}^{-1}=y_{5} y_{2} y_{2} y_{5}^{-1}=1 .
\end{aligned}
$$

If we let $a, b, x$ and $w$ be the images of $a_{1}, b_{1}, x_{1}$ and $x_{2}$ under the epimorphism $\theta: \Gamma \rightarrow G$, then $G$ has partial monodromy presentation

$$
G=\left\langle a, b, x, w \mid x^{t}=w^{t}=x w[a, b]=\cdots=1\right\rangle .
$$

Since the relation $x w[a, b]=1$ makes the generator $w$ redundant, this presentation can be simplified to $G=\left\langle a, b, x \mid x^{t}=([a, b] x)^{t}=\cdots=1\right\rangle$.

Next, if $\theta$ can be extended to a smooth epimorphism $\theta^{\prime}: \Gamma^{\prime} \rightarrow G^{\prime}$ where $G^{\prime}$ is a group containing $G$ as a (normal) subgroup of index 2 , then, letting $\alpha$ be the image 
of $y_{4}$ under $\theta^{\prime}$, we find that

$$
\begin{aligned}
& \theta^{\prime}\left(y_{1}\right)=x^{-1} \alpha b^{-1} x, \quad \theta^{\prime}\left(y_{2}\right)=x^{-1} b \alpha a^{-1}, \\
& \theta^{\prime}\left(y_{3}\right)=a \alpha, \quad \theta^{\prime}\left(y_{4}\right)=\alpha, \quad \theta^{\prime}\left(y_{5}\right)=x,
\end{aligned}
$$

all but the fifth of which lie in $G^{\prime} \backslash G$. Further, conjugation by $\alpha$ is an automorphism of $G$ of order 1 or 2 which acts as follows:

$$
\alpha: \quad a \mapsto a^{-1}, \quad b \mapsto b^{-1}, \quad x \mapsto(a b)^{-1} x^{-1}(b a) .
$$

As in the preceding case, the converse also holds: if $G$ admits such a group automorphism, then $\theta$ can be extended to $\theta^{\prime}: \Gamma^{\prime} \rightarrow G \rtimes_{\alpha} C_{2}$ with $\operatorname{ker} \theta=\operatorname{ker} \theta^{\prime}=\Lambda$, and so $G$ is not the full automorphism group of $X$.

Case N3: $\quad \sigma(\Gamma)=(1 ; t) ; \quad \sigma\left(\Gamma^{\prime}\right)=(0 ; 2,2,2,2 t) ;\left|\Gamma^{\prime}: \Gamma\right|=2$.

In this case the defining relations for $\Gamma$ are $x_{1}^{t}=x_{1}\left[a_{1}, b_{1}\right]=1$, or simply $\left[a_{1}, b_{1}\right]^{t}=1$ (noting that the generator $x_{1}$ is redundant), while those for $\Gamma^{\prime}$ are $y_{1}^{2}=y_{2}^{2}=y_{3}^{2}=y_{4}^{2 t}=y_{1} y_{2} y_{3} y_{4}=1$, and an embedding of $\Gamma$ in $\Gamma^{\prime}$ is given by $a_{1} \mapsto y_{1} y_{2}$ and $b_{1} \mapsto y_{3} y_{2}$ (and $x_{1} \mapsto y_{4}^{2}$ ).

Let $a, b$ and $x$ denote the images of $a_{1}, b_{1}$ and $x_{1}$ under the epimorphism $\theta: \Gamma \rightarrow$ $G$. If $\theta$ can be extended to a smooth epimorphism $\theta^{\prime}: \Gamma^{\prime} \rightarrow G^{\prime}$ where $\left|G^{\prime}: G\right|=2$, then, letting $\alpha=\theta^{\prime}\left(y_{2}\right)$, we have

$$
\theta^{\prime}\left(y_{1}\right)=a \alpha, \quad \theta^{\prime}\left(y_{2}\right)=\alpha, \quad \theta^{\prime}\left(y_{3}\right)=\alpha b^{-1}, \quad \theta^{\prime}\left(y_{4}\right)=b \alpha a^{-1},
$$

and conjugation by $\alpha$ gives the following automorphism of $G$ :

$$
\left.\alpha: \quad a \mapsto a^{-1}, \quad b \mapsto b^{-1} \quad \text { (and } x \mapsto(b a)^{-1} x(b a)\right) .
$$

Conversely, the existence of such an involutory automorphism prevents $G$ from being the full automorphism group of $X$.

Case N4: $\quad \sigma(\Gamma)=(0 ; t, t, t, t), t \geq 3 ; \quad \sigma\left(\Gamma^{\prime}\right)=(0 ; 2,2,2, t) ;\left|\Gamma^{\prime}: \Gamma\right|=4$.

Here an embedding of $\Gamma$ in $\Gamma^{\prime}$ is given by $x_{1} \mapsto y_{2} y_{4} y_{2}, \quad x_{2} \mapsto y_{3} y_{2} y_{4} y_{2} y_{3}$, $x_{3} \mapsto\left(y_{3} y_{2} y_{4}^{-1} y_{2}\right) y_{4}\left(y_{2} y_{4} y_{2} y_{3}\right)$ and $x_{4} \mapsto y_{4}$.

Let $a, b, c$ and $d$ denote the images of $x_{1}, x_{2}, x_{3}$ and $x_{4}$ under the smooth epimorphism $\theta: \Gamma \rightarrow G$. If $\theta$ can be extended to a smooth epimorphism $\theta^{\prime}: \Gamma^{\prime} \rightarrow G^{\prime}$ where $\left|G^{\prime}: G\right|=4$, then, letting $\alpha=\theta^{\prime}\left(y_{3}\right)$ and $\beta=\theta^{\prime}\left(y_{2}\right)$, we have

$$
\theta^{\prime}\left(y_{1}\right)=d^{-1} \alpha \beta, \quad \theta^{\prime}\left(y_{2}\right)=\beta, \quad \theta^{\prime}\left(y_{3}\right)=\alpha, \quad \theta^{\prime}\left(y_{4}\right)=d .
$$

Note that $\alpha$ and $\beta$ are elements of order 2 in $G^{\prime} \backslash G$, with commutator $[\alpha, \beta]=$ $\theta^{\prime}\left(\left[y_{3}, y_{2}\right]\right)=\theta^{\prime}\left(y_{3} y_{2} y_{4} y_{1}\right)=\theta^{\prime}\left(y_{3} y_{2} y_{4} y_{2} y_{3} y_{4}\right)=\theta\left(x_{2} x_{4}\right)=b d \in G$. Further, conjugation by each of $\alpha$ and $\beta$ gives the following two automorphisms of $G$ of order 1 or 2 :

$$
\begin{aligned}
& \alpha: \quad a \mapsto b, \quad b \mapsto a, \quad c \mapsto a^{-1} d a, \quad d \mapsto b c b^{-1}, \\
& \beta: \quad a \mapsto d, \quad b \mapsto d^{-1} c d, \quad c \mapsto a b a^{-1}, \quad d \mapsto a .
\end{aligned}
$$

Conversely, if $G$ admits such automorphisms, then $G$ is not the full automorphism group of $X$, for in this case the action of $G$ extends to an action of its index 4 extension $G^{\prime}=\left(G \rtimes_{\alpha} C_{2}\right) \rtimes_{\beta} C_{2}$, generated by $G$ and elements $\alpha$ and $\beta$ of order 2 which act on $G$ as above and satisfy $\beta \alpha \beta=\alpha b d=d^{-1} b^{-1} \alpha$. In fact, as we will see in case N5 below (taking $t=u$ ), the existence of an automorphism as induced by $\alpha$ on its own is sufficient to prevent $G$ from being the full automorphism group of $X$. Case N5: $\sigma(\Gamma)=(0 ; t, t, u, u), t+u \geq 5 ; \quad \sigma\left(\Gamma^{\prime}\right)=(0 ; 2,2, t, u) ;\left|\Gamma^{\prime}: \Gamma\right|=2$.

This case is similar to case N4, but with an embedding of $\Gamma$ in $\Gamma^{\prime}$ given by $x_{1} \mapsto y_{2} y_{3} y_{2}, x_{2} \mapsto y_{3}, x_{3} \mapsto y_{3}^{-1} y_{2} y_{4} y_{2} y_{3}$ and $x_{4} \mapsto y_{4}$, and the requirements on 
the extension $\theta^{\prime}: \Gamma^{\prime} \rightarrow G^{\prime}$ (with $\left|G^{\prime}: G\right|=2$ ) being

$$
\theta^{\prime}\left(y_{1}\right)=d^{-1} b^{-1} \alpha, \quad \theta^{\prime}\left(y_{2}\right)=\alpha, \quad \theta^{\prime}\left(y_{3}\right)=b, \quad \theta^{\prime}\left(y_{4}\right)=d
$$

for some involution $\alpha \in G^{\prime} \backslash G$. Conjugation by $\alpha$ gives the following automorphism of $G$ (which is exactly the same automorphism $\alpha$ as in the case N4 when $t=u$ ):

$$
\alpha: \quad a \mapsto b, \quad b \mapsto a, \quad c \mapsto a^{-1} d a, \quad d \mapsto b c b^{-1} .
$$

Conversely, if $G$ admits such an automorphism, then $G \neq$ Aut $X$.

Case N6: $\quad \sigma(\Gamma)=(0 ; t, t, t), t \geq 4 ; \quad \sigma\left(\Gamma^{\prime}\right)=(0 ; 3,3, t) ; \quad\left|\Gamma^{\prime}: \Gamma\right|=3$.

Here an embedding of $\Gamma$ in $\Gamma^{\prime}$ is given by $x_{1} \mapsto y_{2} y_{3} y_{2}^{-1}, \quad x_{2} \mapsto y_{2}^{-1} y_{3} y_{2}$ and $x_{3} \mapsto y_{3}$. Let $a, b$ and $c$ denote the images of $x_{1}, x_{2}$ and $x_{3}$ under the epimorphism $\theta: \Gamma \rightarrow G$. If $\theta$ can be extended to a smooth epimorphism $\theta^{\prime}: \Gamma^{\prime} \rightarrow G^{\prime}$ where $\left|G^{\prime}: G\right|=3$, then, letting $\alpha=\theta^{\prime}\left(y_{2}\right)$, we see that

$$
\theta^{\prime}\left(y_{1}\right)=(\alpha c)^{-1}, \quad \theta^{\prime}\left(y_{2}\right)=\alpha, \quad \theta^{\prime}\left(y_{3}\right)=c,
$$

and conjugation by $\alpha$ gives the following automorphism of $G$ :

$$
\alpha: \quad a \mapsto b, \quad b \mapsto c, \quad c \mapsto a .
$$

Conversely, if $G$ admits such an automorphism (of order dividing 3), then $G$ is not the full automorphism group of $X$.

Case N7: $\sigma(\Gamma)=(0 ; t, t, t), t \geq 4 ; \quad \sigma\left(\Gamma^{\prime}\right)=(0 ; 2,3,2 t) ;\left|\Gamma^{\prime}: \Gamma\right|=6$.

An embedding of $\Gamma$ in $\Gamma^{\prime}$ is given by $x_{1} \mapsto y_{2} y_{3}^{2} y_{2}^{-1}, x_{2} \mapsto y_{2}^{-1} y_{3}^{2} y_{2}$ and $x_{3} \mapsto y_{3}^{2}$. Let $a, b$ and $c$ be the images of $x_{1}, x_{2}$ and $x_{3}$ under the epimorphism $\theta: \Gamma \rightarrow G$. If $\theta$ can be extended to a smooth epimorphism $\theta^{\prime}: \Gamma^{\prime} \rightarrow G^{\prime}$ where $\left|G^{\prime}: G\right|=6$, then, taking $\alpha=\theta^{\prime}\left(y_{2}\right)$ and $\beta=\theta^{\prime}\left(y_{1}\right)$, we have

$$
\theta^{\prime}\left(y_{1}\right)=\beta, \quad \theta^{\prime}\left(y_{2}\right)=\alpha, \quad \theta^{\prime}\left(y_{3}\right)=(\beta \alpha)^{-1} .
$$

Note that $\alpha$ and $\beta$ are elements of $G^{\prime} \backslash G$ of orders 3 and 2 respectively, and $(\beta \alpha)^{2}=\theta^{\prime}\left(\left(y_{1} y_{2}\right)^{2}\right)=\theta^{\prime}\left(y_{3}^{-2}\right)=c^{-1} \in G$, so that $\beta \alpha \beta=c^{-1} \alpha^{-1}$. Further, conjugation by each of $\alpha$ and $\beta$ gives the following two automorphisms of $G$ :

$$
\begin{aligned}
\alpha: & a \mapsto b, & b \mapsto c, & & c \mapsto a . \\
\beta: & a \mapsto c, & b \mapsto c^{-1} b c, & c & \mapsto a .
\end{aligned}
$$

Conversely, if $G$ admits such automorphisms, then $G$ is not the full automorphism group of $X$, for in this case the action of $G$ extends to an action of its index 6 extension $G^{\prime}=\left(G \rtimes_{\alpha} C_{3}\right) \rtimes_{\beta} C_{2}$ generated by $G$ and elements $\alpha$ and $\beta$ of orders 3 and 2 which act on $G$ as above and satisfy $\beta \alpha \beta=c^{-1} \alpha^{-1}$. Of course (as in case N6 above) the existence of the automorphism $\alpha$ on its own is sufficient to prevent $G$ from being the full automorphism group of $X$. Similarly, the existence of the automorphism $\beta$ on its own prevents $G$ from being the full group, as we will see in case N8 below (with $t=u$ ).

Case N8: $\quad \sigma(\Gamma)=(0 ; t, t, u), t \geq 3, t+u \geq 7 ; \quad \sigma\left(\Gamma^{\prime}\right)=(0 ; 2, t, 2 u) ; \quad\left|\Gamma^{\prime}: \Gamma\right|=2$.

This case is similar to case $N 6$, but with an embedding of $\Gamma$ in $\Gamma^{\prime}$ given by $x_{1} \mapsto y_{3}^{-1} y_{2} y_{3}, \quad x_{2} \mapsto y_{2}$ and $x_{3} \mapsto y_{3}^{2}$, and the requirements on the extension $\theta^{\prime}: \Gamma^{\prime} \rightarrow G^{\prime}$ (with $\left|G^{\prime}: G\right|=2$ ) being

$$
\theta^{\prime}\left(y_{1}\right)=\alpha, \quad \theta^{\prime}\left(y_{2}\right)=b, \quad \theta^{\prime}\left(y_{3}\right)=(\alpha b)^{-1},
$$

for some involution $\alpha \in G^{\prime} \backslash G$. Conjugation by $\alpha$ gives the following automorphism of $G$ :

$$
\alpha: \quad a \mapsto b, \quad b \mapsto a, \quad c \mapsto b c b^{-1}
$$


Note that after a cyclic permutation of the generators, this is exactly the same automorphism as the automorphism $\beta$ in case N7 (with $u=t$ ).

Conversely, if $G$ admits such an automorphism, then $G \neq \operatorname{Aut} X$.

\section{Non-NORMal EXtensions}

In this section we deal with those cases from Singerman's list in Table 1 for which $\Gamma$ is a non-normal subgroup of the group $\Gamma^{\prime}$. We adopt the same notation as in the previous section for signature presentations of $\Gamma$ and $\Gamma^{\prime}$, and also largely the same approach, except that the emphasis shifts (by necessity) from extensions by group automorphisms to other types of group extensions. The fundamentals, however, remain the same: if the smooth epimorphism $\theta: \Gamma \rightarrow G$ can be extended to another smooth epimorphism $\theta^{\prime}$ from $\Gamma^{\prime}$ onto some group $G^{\prime}$ containing $G$ as a subgroup of index $\left|\Gamma^{\prime}: \Gamma\right|$, then $G^{\prime}$ is (isomorphic to) a group of automorphisms of $X$ larger than $G$, so that $G$ is not the full automorphism group of $X$.

Many of the calculations are more technical than those in Section [3 but can easily be carried out with the assistance of the MAGMA system [2].

Case T1: $\sigma(\Gamma)=(0 ; 7,7,7) ; \quad \sigma\left(\Gamma^{\prime}\right)=(0 ; 2,3,7) ;\left|\Gamma^{\prime}: \Gamma\right|=24$.

In this case the defining relations for the group $\Gamma$ are $x_{1}^{7}=x_{2}^{7}=x_{3}^{7}=x_{1} x_{2} x_{3}=1$, while those for $\Gamma^{\prime}$ are $y_{1}^{2}=y_{2}^{3}=y_{3}^{7}=y_{1} y_{2} y_{3}=1$, and an embedding of $\Gamma$ in $\Gamma^{\prime}$ is given by

$$
\begin{aligned}
& x_{1} \mapsto\left(y_{2}^{-1} y_{3}^{2} y_{2} y_{3}^{-2} y_{2}\right) y_{3}\left(y_{2}^{-1} y_{3}^{2} y_{2} y_{3}^{-2} y_{2}\right)^{-1}, \\
& x_{2} \mapsto\left(y_{2}^{-1} y_{3}^{2} y_{2} y_{3}^{-2} y_{2}\right)^{-1} y_{3}\left(y_{2}^{-1} y_{3}^{2} y_{2} y_{3}^{-2} y_{2}\right), \\
& x_{3} \mapsto y_{3} .
\end{aligned}
$$

If $a, b$ and $c$ are the images of $x_{1}, x_{2}$ and $x_{3}$ under the epimorphism $\theta: \Gamma \rightarrow G$, then $G$ has partial monodromy presentation $G=\langle a, b, c| a^{7}=b^{7}=c^{7}=a b c=$ $\cdots=1\rangle$. If $\theta$ can be extended to a smooth epimorphism $\theta^{\prime}: \Gamma^{\prime} \rightarrow G^{\prime}$ where $\left|G^{\prime}: G\right|=24$, then, letting $\alpha=\theta^{\prime}\left(y_{2}\right)$, we have $\theta^{\prime}\left(y_{1}\right)=(\alpha c)^{-1}, \theta^{\prime}\left(y_{2}\right)=\alpha$, and $\theta^{\prime}\left(y_{3}\right)=c$, the first two of which lie in $G^{\prime} \backslash G$, while the third lies in $G$. In this case, however, $\alpha$ does not normalise $G$ (although the above embedding of $\Gamma$ in $\Gamma^{\prime}$ shows that the element $\alpha^{-1} c^{2} \alpha c^{-2} \alpha=\theta^{\prime}\left(y_{2}^{-1} y_{3}^{2} y_{2} y_{3}^{-2} y_{2}\right)$ of order 3 does, as in the case N6 in the previous section). Instead, the group $G^{\prime}$ contains $G$ as a non-normal subgroup, of index 24.

Now the $(2,3,7)$ triangle group $\Gamma^{\prime}$ has only one conjugacy class of subgroups of index 24 , a fact which may be verified easily by considering transitive permutation representations of $\Gamma^{\prime}$ of degree 24, or with the help of the low-index-subgroups procedure in Magma. Further, the natural permutation representation of $\Gamma^{\prime}$ induced by right multiplication on right cosets of any such subgroup may be given by

$$
\begin{array}{ccc}
y_{1} & \mapsto \quad(1,3)(2,4)(5,7)(6,8)(9,11)(10,12)(13,15)(14,16)(17,19) \\
& (18,20)(21,23)(22,24) \\
y_{2} & \mapsto \quad & (1,2,3)(4,5,6)(7,23,15)(8,16,24)(9,10,11)(12,13,14) \\
& (17,18,19)(20,21,22), \\
y_{3} \quad \mapsto \quad & (2,3,4,8,22,23,5)(6,7,13,10,11,12,16)(14,15,21,18,19,20,24) .
\end{array}
$$

These three permutations generate a group of order 168, which is isomorphic to $\operatorname{PSL}(2,7)$, the automorphism group of Klein's quartic surface of genus 3 . In particular, assigning to the generators $y_{1}, y_{2}$ and $y_{3}$ the linear fractional transformations $z \mapsto-1 / z, \quad z \mapsto(z-1) / z$ and $z \mapsto z-1$ (over $\mathrm{GF}(7)$ ) provides a surjective homomorphism from $\Gamma^{\prime}$ onto $\operatorname{PSL}(2,7)$, under which the images of $x_{1}, x_{2}$ and $x_{3}$ 
are the transformations $z \mapsto z-2, \quad z \mapsto z-4$ and $z \mapsto z-1$ respectively. The kernel of this homomorphism is a surface Fuchsian group of signature $(3 ;-)$, of index 7 in $\Gamma$ and index 168 in $\Gamma^{\prime}$, and generated by conjugates of

$$
\left(y_{2} y_{1} y_{3}\right)^{4}=\left(y_{2} y_{1} y_{2}^{-1} y_{1}\right)^{4}=x_{2} x_{3}^{3}=x_{1}^{-1} x_{3}^{2} \text {. }
$$

It follows that if the epimorphism $\theta: \Gamma \rightarrow G$ can be extended to a smooth epimorphism $\theta^{\prime}: \Gamma^{\prime} \rightarrow G^{\prime}$ where $\left|G^{\prime}: G\right|=24$, then also $G^{\prime}$ has the same transitive permutation representation on cosets of $G$, with kernel $K$ of index 7 in $G$ and index 168 in $G^{\prime}$, generated by conjugates of $\theta\left(x_{2} x_{3}^{3}\right)=b c^{3}=a^{-1} c^{2}$. Note that $G / K \cong C_{7}$, while $G^{\prime} / K \cong \operatorname{PSL}(2,7)$.

Conversely, suppose $G$ has such a normal subgroup $K$ (of index 7 and generated by conjugates of $b c^{3}=a^{-1} c^{2}$ ), and $G$ is extendable to a group $G^{\prime}$ containing $G$ as a subgroup of index 24 and generated by $c$ and an element $\alpha$ such that $\alpha$ normalises $K$ and also satisfies $\alpha^{3}=1,(\alpha c)^{2}=1, a=\left(\alpha^{-1} c^{2} \alpha c^{-2} \alpha\right) c\left(\alpha^{-1} c^{2} \alpha c^{-2} \alpha\right)^{-1}$ and $b=\left(\alpha^{-1} c^{2} \alpha c^{-2} \alpha\right)^{-1} c\left(\alpha^{-1} c^{2} \alpha c^{-2} \alpha\right)$. Then the smooth epimorphism $\theta: \Gamma \rightarrow G$ can be extended to a smooth epimorphism $\theta^{\prime}: \Gamma^{\prime} \rightarrow G^{\prime}$ with $\operatorname{ker} \theta^{\prime}=\operatorname{ker} \theta=\Lambda$. So $G^{\prime} \cong \Gamma^{\prime} / \Lambda$ is a group of automorphisms of $X=U / \Lambda$ larger than $G$, and $G \neq$ Aut $X$.

In fact, it can be seen from case N6 above that the existence of the element $\alpha^{-1} c^{2} \alpha c^{-2} \alpha$ (which induces an automorphism of $G$ of order 3 given by $a \mapsto b \mapsto$ $c \mapsto a$ ) on its own is sufficient to prevent $G$ from being the full automorphism group of $X$.

Case T2: $\quad \sigma(\Gamma)=(0 ; 2,7,7) ; \quad \sigma\left(\Gamma^{\prime}\right)=(0 ; 2,3,7) ; \quad\left|\Gamma^{\prime}: \Gamma\right|=9$.

This case is very similar to the previous one. An embedding of $\Gamma$ in $\Gamma^{\prime}$ is given by $x_{1} \mapsto y_{1}, x_{2} \mapsto\left(y_{2} y_{1} y_{2}\right)^{-1} y_{3}^{-1}\left(y_{2} y_{1} y_{2}\right)$ and $x_{3} \mapsto\left(y_{2} y_{1} y_{2}^{-1}\right) y_{3}^{-1}\left(y_{2} y_{1} y_{2}^{-1}\right)$. The natural permutation representation induced by $\Gamma^{\prime}$ on cosets of $\Gamma$ is given by $y_{1} \mapsto$ $(2,4)(3,7)(5,6)(8,9), \quad y_{2} \mapsto(1,2,3)(4,5,6)(7,8,9)$, and $y_{3} \mapsto(1,7,8,3,4,5,2)$. These permutations generate a group of order 504, isomorphic to the group $\operatorname{PSL}(2,8)$ in its natural action on the projective line over GF(8). The kernel of the representation is a surface Fuchsian group of signature $(7 ;-)$, of index 56 in $\Gamma$ and index 504 in $\Gamma^{\prime}$, and generated by conjugates of

$$
\left(y_{1} y_{3}^{-2} y_{1} y_{3}^{3} y_{1} y_{3}^{3}\right)^{2}=\left(y_{2} y_{1} y_{2} y_{1} y_{2}^{-1} y_{1} y_{2}^{-1} y_{1} y_{2} y_{1} y_{2}^{-1} y_{1} y_{2}^{-1} y_{1}\right)^{2}=\left(x_{2} x_{3}^{-1} x_{2} x_{1} x_{3}^{3}\right)^{-1} \text {. }
$$

If $\theta$ can be extended to $\theta^{\prime}: \Gamma^{\prime} \rightarrow G^{\prime}$ where $\left|G^{\prime}: G\right|=9$, then, letting $\alpha=\theta^{\prime}\left(y_{2}\right)$, we require $\theta^{\prime}\left(y_{1}\right)=a, \quad \theta^{\prime}\left(y_{2}\right)=\alpha$, and $\theta^{\prime}\left(y_{3}\right)=(a \alpha)^{-1}$, and the kernel $K$ of the corresponding permutation representation of $G^{\prime}$ on cosets of $G$ is generated by conjugates of $\theta\left(x_{2} x_{3}^{-1} x_{2} x_{1} x_{3}^{3}\right)=b c^{-1} b a c^{3}$, with quotient $G / K$ isomorphic to a semi-direct product $C_{2}^{3} \rtimes C_{7}$ of the elementary abelian group of order 8 by the cyclic group of order 7 , while $G^{\prime} / K \cong \operatorname{PSL}(2,8)$.

Conversely, suppose $G$ has such a normal subgroup $K$ (of index 56 and generated by conjugates of $b c^{-1} b a c^{3}$ ), and $G$ is extendable to a group $G^{\prime}$ containing $G$ as a subgroup of index 9 and generated by $a$ and an element $\alpha$ such that $\alpha$ normalises $K$ and satisfies $\alpha^{3}=1,(a \alpha)^{7}=1, b=(\alpha a \alpha)^{-1} a \alpha(\alpha a \alpha)$ and $c=\left(\alpha a \alpha^{-1}\right) a \alpha\left(\alpha a \alpha^{-1}\right)$. Then the action of $G$ can be extended to one of $G^{\prime}$ as a larger group of automorphisms of $X=U / \Lambda$.

Case T3: $\quad \sigma(\Gamma)=(0 ; 3,3,7) ; \quad \sigma\left(\Gamma^{\prime}\right)=(0 ; 2,3,7) ; \quad\left|\Gamma^{\prime}: \Gamma\right|=8$.

This case is again similar to case $\mathrm{T} 1$, indeed may be viewed as part of it. An embedding of $\Gamma$ in $\Gamma^{\prime}$ is given by $x_{1} \mapsto y_{2} y_{3}^{-2} y_{2} y_{3}^{2} y_{2}^{-1}, x_{2} \mapsto y_{2}^{-1} y_{3}^{2} y_{2} y_{3}^{-2} y_{2}$ and $x_{3} \mapsto y_{3}$. The permutation representation induced by $\Gamma^{\prime}$ on cosets of $\Gamma$ is given by $y_{1} \mapsto(1,3)(2,4)(5,7)(6,8), y_{2} \mapsto(1,2,3)(4,5,6)$ and $y_{3} \mapsto(2,3,4,8,6,7,5)$, with 
these permutations again generating a group of order 168 isomorphic to $\operatorname{PSL}(2,7)$, here in its natural action on the projective line over $\operatorname{GF}(7)$. The kernel of this representation is the same surface group of signature $(3 ;-)$ as in case $\mathrm{T} 1$, generated by conjugates of $\left(y_{2} y_{1} y_{3}\right)^{4}=\left(y_{2} y_{1} y_{2}^{-1} y_{1}\right)^{4}=x_{2} x_{1} x_{3}^{2}=x_{1}^{-1} x_{3}^{-1} x_{1} x_{3}^{2}$, but now of index 21 in $\Gamma$.

If $\theta$ can be extended to $\theta^{\prime}: \Gamma^{\prime} \rightarrow G^{\prime}$ where $\left|G^{\prime}: G\right|=8$, then, letting $\alpha=\theta^{\prime}\left(y_{2}\right)$, we require $\theta^{\prime}\left(y_{1}\right)=(\alpha c)^{-1}, \theta^{\prime}\left(y_{2}\right)=\alpha$, and $\theta^{\prime}\left(y_{3}\right)=c$; this time $K$ is generated by conjugates of $\theta\left(x_{2} x_{1} x_{3}^{2}\right)=b a c^{2}=a^{-1} c^{-1} a c^{2}$, with $G / K$ isomorphic to $C_{7} \rtimes C_{3}$, while $G^{\prime} / K \cong \operatorname{PSL}(2,7)$. Conversely, if $G$ has such a normal subgroup $K$ of index 21 , and $G$ is extendable to a group $G^{\prime}$ such that $\left|G^{\prime}: G\right|=8$ and $G^{\prime}$ is generated by $c$ and an element $\alpha$ such that $\alpha$ normalises $K$ and satisfies $\alpha^{3}=1,(\alpha c)^{2}=1$, $a=\alpha c^{-2} \alpha c^{2} \alpha^{-1}$ and $b=\alpha^{-1} c^{2} \alpha c^{-2} \alpha$, then the action of $G$ can be extended to one of $G^{\prime}$ on $X$.

Case T4: $\quad \sigma(\Gamma)=(0 ; 4,8,8) ; \quad \sigma\left(\Gamma^{\prime}\right)=(0 ; 2,3,8) ;\left|\Gamma^{\prime}: \Gamma\right|=12$.

This is again similar to T1, but with an embedding of $\Gamma$ in $\Gamma^{\prime}$ given by $x_{1} \mapsto y_{3}^{2}$, $x_{2} \mapsto\left(y_{2} y_{3}^{-1} y_{2}\right) y_{3}\left(y_{2}^{-1} y_{3} y_{2}^{-1}\right)$ and $x_{3} \mapsto\left(y_{2}^{-1} y_{3}^{-2} y_{2}\right) y_{3}\left(y_{2}^{-1} y_{3}^{2} y_{2}\right)$, and permutation representation of $\Gamma^{\prime}$ on cosets of $\Gamma$ given by

$$
\begin{aligned}
& y_{1} \mapsto(1,9)(2,4)(3,7)(5,6)(8,10)(11,12), \\
& y_{2} \mapsto(1,2,3)(4,5,6)(7,8,9)(10,11,12), \\
& y_{3} \mapsto(1,7)(2,9,10,11,8,3,4,5),
\end{aligned}
$$

These permutations generate a (soluble) group of order 96, which is a non-split extension of a Klein 4-group by the symmetric group $S_{4}$, with the kernel being of index 8 in $\Gamma$ and index 96 in $\Gamma^{\prime}$, and generated by conjugates of $\left(y_{2} y_{1} y_{3}\right)^{3}=$ $\left(y_{2} y_{1} y_{2}^{-1} y_{1}\right)^{3}=x_{2}^{-2} x_{1}=x_{2}^{-3} x_{3}^{-1}$. Also $G / K \cong C_{8}$, while $G^{\prime} / K$ is a non-split extension of $C_{2}^{2}$ by $S_{4}$.

Accordingly, if $G$ has such a normal subgroup $K$ (of index 8 and generated by conjugates of $a^{-1} b^{2}$ ) and $G$ is extendable to a group $G^{\prime}$ with $\left|G^{\prime}: G\right|=12$ and $G^{\prime}$ is generated by elements $\alpha$ and $\beta$ which satisfy $\alpha^{3}=\beta^{8}=(\alpha \beta)^{2}=1$, $a=\beta^{2}, b=\left(\alpha \beta^{-1} \alpha\right) \beta\left(\alpha^{-1} \beta \alpha^{-1}\right)$ and $c=\left(\alpha^{-1} \beta^{-2} \alpha\right) \beta\left(\alpha^{-1} \beta^{2} \alpha\right)$, then the action of $G$ can be extended to one of $G^{\prime}$ on $X$. In fact, from case N8 in Section 3 the existence of the element $\alpha^{-1} \beta^{4} \alpha$ (which induces an involutory automorphism of $G$ interchanging $b$ and $c$ while conjugating $a$ to $c a c^{-1}$ ) is sufficient to prevent $G$ from being the full automorphism group of $X$.

Case T5: $\quad \sigma(\Gamma)=(0 ; 3,8,8) ; \quad \sigma\left(\Gamma^{\prime}\right)=(0 ; 2,3,8) ; \quad\left|\Gamma^{\prime}: \Gamma\right|=10$.

This is again similar to previous cases, but with an embedding of $\Gamma$ given by $x_{1} \mapsto y_{2} y_{3}^{-2} y_{2} y_{3}^{2} y_{2}^{-1}, x_{2} \mapsto y_{2}^{-1} y_{3}^{2} y_{2}^{-1} y_{3} y_{2} y_{3}^{-2} y_{2}$ and $x_{3} \mapsto y_{3}$, and the permutation representation given by

$$
\begin{aligned}
& y_{1} \mapsto(1,3)(2,4)(5,10)(6,7)(8,9), \\
& y_{2} \mapsto(1,2,3)(4,5,6)(7,8,9), \\
& y_{3} \mapsto(2,3,4,7,8,6,10,5) .
\end{aligned}
$$

These permutations generate a group of order 720 , isomorphic to $\operatorname{PGL}(2,9)$, and the kernel is generated by conjugates of $\left(y_{2} y_{1} y_{3}\right)^{5}=\left(y_{2} y_{1} y_{2}^{-1} y_{1}\right)^{5}=x_{2}^{2} x_{1} x_{3}^{2}$ and $\left(y_{3}^{3} y_{2} y_{3}^{-1} y_{1} y_{3}^{2} y_{2} y_{1}\right)^{2}=y_{2}^{-1}\left(x_{3}^{-1} x_{2} x_{1}^{-1} x_{2}^{-1} x_{1} x_{2}^{-1}\right) y_{2}$. Also $G / K \cong C_{3}^{2} \rtimes C_{8}$ and $G^{\prime} / K \cong \operatorname{PGL}(2,9)$.

Hence if $G$ has such a normal subgroup $K$ (of index 72 and generated by conjugates of $b^{2} a c^{2}$ and $\left.c^{-1} b a^{-1} b^{-1} a b^{-1}\right)$ and $G$ is extendable to a group $G^{\prime}$ containing 
$G$ as a subgroup of index 10 and generated by $c$ and an element $\alpha$ such that $\alpha^{3}=1$, $(\alpha c)^{2}=1, a=\alpha c^{-2} \alpha c^{2} \alpha^{-1}$ and $b=\alpha^{-1} c^{2} \alpha^{-1} c \alpha c^{-2} \alpha$, then the action of $G$ can be extended to one of $G^{\prime}$ on $X$; so $G \neq$ Aut $X$.

Case T6: $\quad \sigma(\Gamma)=(0 ; 9,9,9) ; \sigma\left(\Gamma^{\prime}\right)=(0 ; 2,3,9) ;\left|\Gamma^{\prime}: \Gamma\right|=12$.

In this case the key features are an embedding of $\Gamma$ in $\Gamma^{\prime}$ given by

$$
x_{1} \mapsto\left(y_{2} y_{3}^{3} y_{2}^{-1}\right) y_{3}\left(y_{2} y_{3}^{-3} y_{2}^{-1}\right), \quad x_{2} \mapsto\left(y_{2} y_{3}^{-3} y_{2}^{-1}\right) y_{3}\left(y_{2} y_{3}^{3} y_{2}^{-1}\right), \quad x_{3} \mapsto y_{3},
$$

and permutation representation

$$
\begin{aligned}
& y_{1} \mapsto(1,3)(2,4)(5,7)(6,10)(8,9)(11,12), \\
& y_{2} \mapsto(1,2,3)(4,5,6)(7,8,9)(10,11,12), \\
& y_{3} \mapsto(2,3,4,10,11,6,7,8,5) .
\end{aligned}
$$

Here the kernel is generated by conjugates of

$$
y_{3}^{-2} y_{2}^{-1} y_{3}^{2} y_{2} y_{3}^{-2} y_{2}^{-1} y_{3} y_{2} y_{3}^{-2} y_{2}^{-1} y_{3} y_{2}^{-1}=x_{3}^{-1} x_{1} x_{3}^{-1} x_{2},
$$

and $G / K$ is an extraspecial group of order 27 , while $G^{\prime} / K \cong C_{3}^{3} \rtimes A_{4}$ (a semi-direct product of the elementary abelian group of order 27 by the alternating group $A_{4}$ ).

Hence if $G$ has such a normal subgroup $K$ (of index 27 and generated by conjugates of $\left.c^{-1} a c^{-1} b\right)$ and $G$ is extendable to a group $G^{\prime}$ with $\left|G^{\prime}: G\right|=12$ and $G^{\prime}$ is generated by $c$ and an $\alpha$ such that $\alpha^{3}=1,(\alpha c)^{2}=1, a=\left(\alpha c^{3} \alpha^{-1}\right) c\left(\alpha c^{3} \alpha^{-1}\right)^{-1}$ and $b=\left(\alpha c^{3} \alpha^{-1}\right)^{-1} c\left(\alpha c^{3} \alpha^{-1}\right)$, then the action of $G$ can be extended to one of $G^{\prime}$ on $X$. In fact, from case N6 in Section 3 the existence of the element $\alpha c^{3} \alpha^{-1}$ (which induces an automorphism of $G$ of order 3 given by $a \mapsto b \mapsto c \mapsto a$ ) is sufficient to prevent $G$ from being the full automorphism group of $X$.

Case T7: $\sigma(\Gamma)=(0 ; 4,4,5) ; \sigma\left(\Gamma^{\prime}\right)=(0 ; 2,4,5) ;\left|\Gamma^{\prime}: \Gamma\right|=6$.

Here the key features are an embedding of $\Gamma$ given by $x_{1} \mapsto y_{2}^{2} y_{3} y_{2} y_{3}^{-1} y_{2}^{2}, x_{2} \mapsto$ $y_{2}^{-1} y_{3} y_{2} y_{3}^{-1} y_{2}$ and $x_{3} \mapsto y_{3}$, a permutation representation $y_{1} \mapsto(1,4)(2,5)(3,6)$, $y_{2} \mapsto(1,2,3,4)$ and $y_{3} \mapsto(2,4,6,3,5)$, a kernel generated by conjugates of $\left(y_{2} y_{1} y_{3}\right)^{3}=\left(y_{2} y_{1} y_{2}^{-1} y_{1}\right)^{3}=x_{1}^{-1} x_{2}^{-1} x_{3}^{2}=x_{1}^{-1} x_{3} x_{1} x_{3}^{2}$, and $G / K \cong C_{5} \rtimes C_{4}$, while $G^{\prime} / K \cong \operatorname{PGL}(2,5)$.

Hence if $G$ has such a normal subgroup $K$ (of index 20 and generated by conjugates of $\left.a^{-1} b^{-1} c^{2}=a^{-1} c a c^{2}\right)$ and $G$ is extendable to a group $G^{\prime}$ with $\left|G^{\prime}: G\right|=6$ and $G^{\prime}$ is generated by $c$ and an element $\alpha$ such that $\alpha^{4}=1,(\alpha c)^{2}=1, a=$ $\alpha^{2} c \alpha c^{-1} \alpha^{2}$ and $b=\alpha^{-1} c \alpha c^{-1} \alpha$, then the action of $G$ can be extended to one of $G^{\prime}$ on $X$.

Case T8: $\quad \sigma(\Gamma)=(0 ; n, 4 n, 4 n), n \geq 2 ; \quad \sigma\left(\Gamma^{\prime}\right)=(0 ; 2,3,4 n) ;\left|\Gamma^{\prime}: \Gamma\right|=6$.

Here the key features are an embedding of $\Gamma$ given by $x_{1} \mapsto y_{2}^{-1} y_{3}^{4} y_{2}$, $x_{2} \mapsto\left(y_{2}^{-1} y_{3}^{-2} y_{2}\right) y_{3}\left(y_{2}^{-1} y_{3}^{2} y_{2}\right)$ and $x_{3} \mapsto y_{3}$, a permutation representation $y_{1} \mapsto$ $(1,3)(2,6)(4,5), \quad y_{2} \mapsto(1,2,3)(4,5,6)$ and $y_{3} \mapsto(2,3,6,5)$, a kernel generated by conjugates of $y_{3}^{4}=x_{3}^{4}$ (or equivalently by conjugates of $x_{1}$ ), and $G / K \cong C_{4}$, while $G^{\prime} / K \cong S_{4}$.

Hence if $G$ has such a normal subgroup $K$ (of index 4 and generated by conjugates of $a$ ) and $G$ is extendable to a group $G^{\prime}$ with $\left|G^{\prime}: G\right|=6$ and $G^{\prime}$ is generated by $c$ and an element $\alpha$ such that $\alpha^{3}=1,(\alpha c)^{2}=1, a=\theta\left(x_{1}\right)=\alpha^{-1} c^{4} \alpha$ and $b=\theta\left(x_{2}\right)=\alpha^{-1} c^{-2} \alpha c \alpha^{-1} c^{2} \alpha$, then the action of $G$ can be extended to one of $G^{\prime}$ on $X$. In fact, from case $\mathrm{N} 8$, the existence of the element $c \alpha(\alpha c)(c \alpha)^{-1}$ (which induces an automorphism of $G$ of order 2 interchanging $b$ and $c$ while conjugating $a$ to $c a c^{-1}$ ) is sufficient to prevent $G$ from being the full automorphism group of $X$. 
Case T9: $\quad \sigma(\Gamma)=(0 ; n, 2 n, 2 n), n \geq 3 ; \quad \sigma\left(\Gamma^{\prime}\right)=(0 ; 2,4,2 n) ; \quad\left|\Gamma^{\prime}: \Gamma\right|=4$.

Here the key features are an embedding of $\Gamma$ in $\Gamma^{\prime}$ given by $x_{1} \mapsto y_{2} y_{3}^{2} y_{2}^{-1}$, $x_{2} \mapsto y_{2}^{2} y_{3} y_{2}^{2}$ and $x_{3} \mapsto y_{3}$, a permutation representation $y_{1} \mapsto(1,4)(2,3)$, $y_{2} \mapsto(1,2,3,4)$, and $y_{3} \mapsto(2,4)$, a kernel generated by conjugates of $y_{3}^{2}=x_{3}^{2}$ (or equivalently by conjugates of $x_{1}$ ), and $G / K \cong C_{2}$, while $G^{\prime} / K \cong D_{4}$ (dihedral of order 8).

Hence if $G$ has such a normal subgroup $K$ (of index 2 and generated by conjugates of $a$ ) and $G$ is extendable to a group $G^{\prime}$ with $\left|G^{\prime}: G\right|=4$ and $G^{\prime}$ is generated by $c$ and an element $\alpha$ such that $\alpha^{4}=1,(\alpha c)^{2}=1, a=\theta\left(x_{1}\right)=\alpha c^{2} \alpha^{-1}$ and $b=\theta\left(x_{2}\right)=\alpha^{2} c \alpha^{2}$, then the action of $G$ can be extended to one of $G^{\prime}$ on $X$. In fact, from case N8 in Section 3, the existence of the element $\alpha^{2}$ (which induces an automorphism of $G$ of order 2 given by $a \mapsto c a c^{-1}$ and $\left.b \mapsto c \mapsto b\right)$ is sufficient to prevent $G$ from being the full automorphism group of $X$.

Case T10: $\quad \sigma(\Gamma)=(0 ; 3, n, 3 n), n \geq 3 ; \quad \sigma\left(\Gamma^{\prime}\right)=(0 ; 2,3,3 n) ; \quad\left|\Gamma^{\prime}: \Gamma\right|=4$.

Here the key features are an embedding of $\Gamma$ given by $x_{1} \mapsto y_{1} y_{3} y_{2} y_{3}^{-1} y_{1}, x_{2} \mapsto$ $y_{1} y_{3}^{3} y_{1}$ and $x_{3} \mapsto y_{3}$, a permutation representation $y_{1} \mapsto(1,3)(2,4), y_{2} \mapsto(1,2,3)$ and $y_{3} \mapsto(2,3,4)$, a kernel generated by conjugates of $y_{3}^{3}=x_{3}^{3}$ (or equivalently by conjugates of $x_{2}$ ), and $G / K \cong C_{3}$, while $G^{\prime} / K \cong A_{4}$.

Hence if $G$ has such a normal subgroup $K$ (of index 3 and generated by conjugates of $b$ ) and $G$ is extendable to a group $G^{\prime}$ with $\left|G^{\prime}: G\right|=4$ and $G^{\prime}$ is generated by $c$ and an element $\alpha$ such that $\alpha^{2}=1,(c \alpha)^{3}=1, a=\theta\left(x_{1}\right)=\alpha c(c \alpha)^{-1} c^{-1} \alpha$ and $b=\theta\left(x_{2}\right)=\alpha c^{3} \alpha$, then the action of $G$ can be extended to one of $G^{\prime}$ on $X$.

Case T11: $\sigma(\Gamma)=(0 ; 2, n, 2 n), n \geq 4 ; \sigma\left(\Gamma^{\prime}\right)=(0 ; 2,3,2 n) ;\left|\Gamma^{\prime}: \Gamma\right|=3$.

In this final case the key features are an embedding of $\Gamma$ in $\Gamma^{\prime}$ given by $x_{1} \mapsto$ $y_{2} y_{1} y_{2}^{-1}, \quad x_{2} \mapsto y_{2}^{-1} y_{3}^{2} y_{2}$ and $x_{3} \mapsto y_{3}$, a permutation representation $y_{1} \mapsto(1,3)$, $y_{2} \mapsto(1,2,3)$ and $y_{3} \mapsto(2,3)$, a kernel generated by conjugates of $y_{3}^{2}=x_{3}^{2}$ (or equivalently by conjugates of $x_{2}$ ), and $G / K \cong C_{2}$, while $G^{\prime} / K \cong S_{3}$. Accordingly, if $G$ has such a normal subgroup $K$ (of index 2 and generated by conjugates of $b$ ) and $G$ is extendable to a group $G^{\prime}$ with $\left|G^{\prime}: G\right|=3$ and $G^{\prime}$ is generated by $c$ and an element $\alpha$ such that $\alpha^{3}=1,(\alpha c)^{2}=1, a=\theta\left(x_{1}\right)=\alpha(\alpha c) \alpha^{-1}$ and $b=\theta\left(x_{2}\right)=\alpha^{-1} c^{2} \alpha$, then the action of $G$ can be extended to one of $G^{\prime}$ on $X$; so $G \neq$ Aut $X$.

\section{Results}

We summarise our results in the following two theorems. The first covers cases N1 to N5, in which the Fuchsian group $\Gamma$ is not a triangle group. As noted earlier, conditions for extendability in case N4 are covered by case N5.

Theorem 5.1. Let $G$ be a finite group acting with a non-maximal and non-triangular Fuchsian signature on a compact Riemann surface $X$ of genus $g$.

(i) If $G$ acts with signature $(2 ;-)$, and for a corresponding presentation $G=$ $\langle a, b, c, d \mid[a, b][c, d]=\cdots=1\rangle$ the assignment $a \mapsto a^{-1}, b \mapsto a b^{-1} a^{-1}, c \mapsto$ $\left(b^{-1} c d\right) c^{-1}\left(b^{-1} c d\right)^{-1}$ and $d \mapsto\left(b^{-1} c\right) d^{-1}\left(b^{-1} c\right)^{-1}$ is an automorphism of $G$, then $G$ is not the full automorphism group of $X$.

(ii) If $G$ acts with signature $(1 ; t, t)$, and for a corresponding presentation $G=$ $\left\langle a, b, x \mid x^{t}=([a, b] x)^{t}=\cdots=1\right\rangle$ the assignment $a \mapsto a^{-1}, b \mapsto b^{-1}$ and $x \mapsto(a b)^{-1} x^{-1}(b a)$ is an automorphism of $G$, then $G$ is not the full automorphism group of $X$. 
(iii) If $G$ acts with signature $(1 ; t)$ and for a corresponding presentation $G=$ $\left\langle a, b \mid[a, b]^{t}=\cdots=1\right\rangle$ the assignment $a \mapsto a^{-1}$ and $b \mapsto b^{-1}$ is an automorphism of $G$, then $G$ is not the full automorphism group of $X$.

(iv) If $G$ acts with signature $(0 ; t, t, u, u)$, where $t+u \geq 5$, and for a corresponding presentation $G=\left\langle a, b, c, d \mid a^{t}=b^{t}=c^{u}=d^{u}=a b c d=\cdots=1\right\rangle$ the assignment $a \mapsto b, b \mapsto a, c \mapsto a^{-1} d a$ and $d \mapsto b c b^{-1}$ is an automorphism of $G$, then $G$ is not the full automorphism group of $X$.

In the preceding theorem the converse is not always true: if $G$ does not admit an automorphism of the appropriate form, then although the epimorphism $\theta: \Gamma \rightarrow G$ cannot be extended to a Fuchsian group $\Gamma^{\prime}$ in the way described in cases N1 to N5, it may still be possible for $\theta$ to be properly extended to another Fuchsian group $\Gamma^{\prime \prime}$ containing $\Gamma$.

On the other hand, for triangle groups no extension is possible unless the group appears in Singerman's list (in Table 1). The reason for this is that all embeddings of an abstract triangle group into $\operatorname{PSL}(2, \mathbb{R})$ are mutually conjugate, since the Teichmüller dimension is zero.

Accordingly, if the action of a finite group $G$ on a surface $X$ with signature $\sigma=\left(0 ; m_{1}, m_{2}, m_{3}\right)$ extends to one of a larger group $G^{\prime}$, then $G^{\prime}$ necessarily acts with signature $\sigma^{\prime}=\left(0 ; m_{1}^{\prime}, m_{2}^{\prime}, m_{3}^{\prime}\right)$, where the pair $\left(\sigma, \sigma^{\prime}\right)$ appears in Singerman's list. Moreover, in this case the canonical generators of $\Gamma^{\prime}$ may be chosen so that the embedding of $\Gamma$ in $\Gamma^{\prime}$ is as given in one of the cases N6 to N8 or T1 to T11.

Again we have a single theorem to deal with all these cases. Note that a group acting with a fixed signature may fulfill different conditions for its extendability, and thus may admit more than one type of extension; in particular, in the theorem below, cases N7, T1 and T6 are covered by case N6, while N7, T4, T8 and T9 are covered by $\mathrm{N} 8$.

Theorem 5.2. Let $G$ be a finite group acting on a compact Riemann surface $X$ of genus $g$ with a triangular signature $\left(0 ; m_{1}, m_{2}, m_{3}\right)$, corresponding to a presentation of the form $G=\left\langle a, b, c \mid a^{m_{1}}=b^{m_{2}}=c^{m_{3}}=a b c=\cdots=1\right\rangle$. Then $G$ is the full automorphism group of $X$ unless at least one of the following conditions is satisfied (up to permutation of the periods $m_{1}, m_{2}, m_{3}$ ), in which case $G \neq$ Aut $X$ :

(i) $G$ acts with signature $(0 ; t, t, t)$ where $t \geq 4$, and the assignment $a \mapsto b$, $b \mapsto c$ and $c \mapsto a$ induces an automorphism of $G$;

(ii) $G$ acts with signature $(0 ; t, t, u)$ where $t \geq 3$ and $t+u \geq 7$, and the assignment $a \mapsto b, b \mapsto a$ and $c \mapsto b c b^{-1}$ induces an automorphism of $G$;

(iii) $G$ acts with signature $(0 ; 2,7,7)$, the conjugates of $b c^{-1} b a c^{3}$ generate a normal subgroup $K$ of index 56 in $G$, and $G$ is extendable to a group $G^{\prime}$ containing $G$ as a subgroup of index 9 such that $G^{\prime}$ is generated by a and an element $\alpha$ which normalises $K$ and satisfies $\alpha^{3}=1,(a \alpha)^{7}=1, b=(\alpha a \alpha)^{-1} a \alpha(\alpha a \alpha)$ and $c=\left(\alpha a \alpha^{-1}\right) a \alpha\left(\alpha a \alpha^{-1}\right)$;

(iv) $G$ acts with signature $(0 ; 3,3,7)$, the conjugates of bac ${ }^{2}$ generate a normal subgroup $K$ of index 21 in $G$, and $G$ is extendable to a group $G^{\prime}$ containing $G$ as a subgroup of index 8 such that $G^{\prime}$ is generated by $c$ and an element $\alpha$ which normalises $K$ and satisfies $\alpha^{3}=1,(\alpha c)^{2}=1, a=\alpha c^{-2} \alpha c^{2} \alpha^{-1}$ and $b=\alpha^{-1} c^{2} \alpha c^{-2} \alpha$;

(v) $G$ acts with signature $(0 ; 3,8,8)$, conjugates of $b^{2} a c^{2}$ and $c^{-1} b a^{-1} b^{-1} a b^{-1}$ generate a normal subgroup $K$ of index 72 in $G$, and $G$ is extendable to a group $G^{\prime}$ containing $G$ as a subgroup of index 10 such that $G^{\prime}$ is generated by $c$ and an 
element $\alpha$ which normalises $K$ and satisfies $\alpha^{3}=1,(\alpha c)^{2}=1, a=\alpha c^{-2} \alpha c^{2} \alpha^{-1}$ and $b=\alpha^{-1} c^{2} \alpha^{-1} c \alpha c^{-2} \alpha$;

(vi) $G$ acts with signature $(0 ; 4,4,5)$, the conjugates of $a^{-1} b^{-1} c^{2}$ generate a normal subgroup $K$ of index 20 in $G$, and $G$ is extendable to a group $G^{\prime}$ containing $G$ as a subgroup of index 6 such that $G^{\prime}$ is generated by $c$ and an element $\alpha$ which normalises $K$ and satisfies $\alpha^{4}=1,(\alpha c)^{2}=1, a=\alpha^{2} c \alpha c^{-1} \alpha^{2}$ and $b=\alpha^{-1} c \alpha c^{-1} \alpha$;

(vii) $G$ acts with signature $(0 ; 3, n, 3 n)$ where $n \geq 3$, the conjugates of $b$ generate a normal subgroup $K$ of index 3 in $G$, and $G$ is extendable to a group $G^{\prime}$ containing $G$ as a subgroup of index 4 such that $G^{\prime}$ is generated by $c$ and an element $\alpha$ which normalises $K$ and satisfies $\alpha^{2}=1,(c \alpha)^{3}=1, a=\alpha c(c \alpha)^{-1} c^{-1} \alpha$ and $b=\alpha c^{3} \alpha$;

(viii) $G$ acts with signature $(0 ; 2, n, 2 n)$ where $n \geq 4$, the conjugates of $b$ generate a normal subgroup $K$ of index 2 in $G$, and $G$ is extendable to a group $G^{\prime}$ containing $G$ as a subgroup of index 3 such that $G^{\prime}$ is generated by $c$ and an element $\alpha$ which normalises $K$ and satisfies $\alpha^{3}=1,(\alpha c)^{2}=1, a=\alpha(\alpha c) \alpha^{-1}$ and $b=\alpha^{-1} c^{2} \alpha$.

At this point we observe also that there are certain arithmetic conditions on the genus $g$ of the surface which are necessary for extendability of the group action as described above, in the cases of non-normal extensions. These conditions (summarised in Table 2) follow from the Riemann-Hurwitz formula and the requirements identified in Section 4

TABLE 2. Arithmetic conditions necessary for non-normal extendability

\begin{tabular}{|c|l|l|}
\hline Case & Conditions on $|G|$ & Conditions on genus $g$ \\
\hline T1 & $|G|=7(g-1) / 2$, divisible by 7 & $g$ odd \\
T2 & $|G|=28(g-1) / 3$, divisible by 56 & $g \equiv 1(\bmod 6)$ \\
T3 & $|G|=21(g-1) / 4$, divisible by 21 & $g \equiv 1(\bmod 4)$ \\
T4 & $|G|=4(g-1)$, divisible by 8 & $g$ odd \\
T5 & $|G|=24(g-1) / 5$, divisible by 72 & $g \equiv 1(\bmod 15)$ \\
T6 & $|G|=3(g-1)$, divisible by 27 & $g \equiv 1(\bmod 9)$ \\
T7 & $|G|=20(g-1) / 3$, divisible by 20 & $g \equiv 1(\bmod 3)$ \\
T8 & $|G|=4 n(g-1) /(2 n-3)$, divisible by 4 & $g \equiv 1\left(\bmod \frac{2 n-3}{\operatorname{gcd}(n, 3)}\right)$ \\
T9 & $|G|=2 n(g-1) /(n-2)$, divisible by 2 & $g \equiv 1\left(\bmod \frac{n-2}{\operatorname{gcd}(n, 2)}\right)$ \\
T10 & $|G|=3 n(g-1) /(n-2)$, divisible by 3 & $g \equiv 1\left(\bmod \frac{n-2}{\operatorname{gcd}(n, 2)}\right)$ \\
T11 & $|G|=4 n(g-1) /(n-3)$, divisible by 2 & $g \equiv 1\left(\bmod \frac{n-3}{\operatorname{gcd}(n-3,6)}\right)$ \\
\hline
\end{tabular}

For example, in case T2, where the signatures of the Fuchsian groups are $\sigma(\Gamma)=$ $(0 ; 2,7,7)$ and $\sigma\left(\Gamma^{\prime}\right)=(0 ; 2,3,7)$, the group $G$ has order $28(g-1) / 3$ and so $g \equiv 1$ (mod 3 ); but further, since $G$ must have a normal subgroup of index $56,|G|$ has to be divisible by 56 , and so $g-1$ is even and thus $g \equiv 1(\bmod 6)$.

\section{Some APplications}

In this section we apply the above results to a few examples, to illustrate the range of possibilities.

Example 6.1. Let $G$ be a cyclic group of order 7 , generated by an element $v$. Up to equivalence there are two smooth homomorphisms from the $(7,7,7)$ triangle group $\Gamma=\left\langle x_{1}, x_{2}, x_{3} \mid x_{1}^{7}=x_{2}^{7}=x_{3}^{7}=x_{1} x_{2} x_{3}=1\right\rangle$ to $G$, each corresponding to 
an action of $G$ on a surface of genus 3 . One of these takes $\left(x_{1}, x_{2}, x_{3}\right)$ to $\left(v, v, v^{5}\right)$, and the other takes $\left(x_{1}, x_{2}, x_{3}\right)$ to $\left(v, v^{2}, v^{4}\right)$.

In the first case, the identity automorphism of $G$ has the effect of interchanging the (coincident) images of $x_{1}$ and $x_{2}$, and so by Theorem 5.2 (ii), we find that $G$ is not the full automorphism group of the associated surface. Indeed, by case N8 the action of $G$ is extendable to the action of a cyclic group $G^{\prime}$ of order 14, generated by $v$ and an involution $\alpha$ which centralises $v$. The latter action corresponds to a smooth homomorphism from the $(2,7,14)$ triangle group onto $G^{\prime}$.

In the second case, the squaring map $v^{k} \rightarrow v^{2 k}$ is an automorphism of $G$ which permutes the images of $x_{1}, x_{2}$ and $x_{3}$ in a 3-cycle. So by Theorem 5.2 (i) we find again that $G$ is not the full automorphism group of the surface. Indeed, by case N6 the action of $G$ is extendable to the action of a semi-direct product $G^{\prime}=C_{7} \rtimes C_{3}$ of order 21, generated by $v$ and an element $\alpha$ such that $\alpha^{3}=1$ and $\alpha^{-1} v \alpha=v^{2}$, corresponding to a smooth homomorphism from the $(3,3,7)$ triangle group onto $G^{\prime}$. (Moreover, by case $\mathrm{T} 1$ the action of $G$ extends to an action of $\operatorname{PSL}(2,7)$, as seen in 3 .)

Example 6.2. Let $G=\langle v\rangle$ be a cyclic group of order 11. Up to equivalence there are two smooth homomorphisms from the $(11,11,11)$ triangle group $\Gamma=$ $\left\langle x_{1}, x_{2}, x_{3} \mid x_{1}^{11}=x_{2}^{11}=x_{3}^{11}=x_{1} x_{2} x_{3}=1\right\rangle$ to $G$, each corresponding to an action of $G$ on a surface of genus 5 . One of these takes $\left(x_{1}, x_{2}, x_{3}\right)$ to $\left(v, v, v^{9}\right)$, and the other takes $\left(x_{1}, x_{2}, x_{3}\right)$ to $\left(v, v^{2}, v^{8}\right)$.

In the first case, the identity automorphism of $G$ has the effect of interchanging the (coincident) images of $x_{1}$ and $x_{2}$. So by Theorem 5.2 (ii), we know that $G$ is not the full automorphism group of the associated surface. Indeed, by case N8 the action of $G$ is extendable to the action of a cyclic group $G^{\prime}$ of order 22 , generated by $v$ and an involution $\alpha$ which centralises $v$, corresponding to a smooth homomorphism from the $(2,11,22)$ triangle group onto $G^{\prime}$.

In the second case, however, $G$ has no such automorphism, and also no automorphism of $G$ which permutes the images of $x_{1}, x_{2}$ and $x_{3}$ in a 3-cycle. Hence by Theorem 5.2 we find that $G$ is the full automorphism group of the surface in this case.

Example 6.3. Let $G=S_{7}$ be the symmetric group of degree 7 . This group acts faithfully on a surface of genus 1681, associated with a smooth homomorphism from the $(3,4,12)$ triangle group $\Gamma=\left\langle x_{1}, x_{2}, x_{3} \mid x_{1}^{3}=x_{2}^{4}=x_{3}^{12}=x_{1} x_{2} x_{3}=1\right\rangle$ to $G$ given by $x_{1} \mapsto(1,2,3)(4,5,6), x_{2} \mapsto(2,7,3,4)$ and $x_{3} \mapsto(1,3,7)(2,6,5,4)$. Since $S_{7}$ has no normal subgroup of index 3 , this homomorphism cannot be extended to a smooth homomorphism from the $(2,3,12)$ triangle group to a group $G^{\prime}$ containing $S_{7}$ as a subgroup of index 4 (as per Theorem [5.2(vii)). Hence $S_{7}$ is the full automorphism group of the surface in this case.

Example 6.4. Let $G=S_{10}$, and consider the actions of $G$ on surfaces of genus 1451521 associated with the following two inequivalent smooth homomorphisms from the $(2,5,10)$ triangle group $\Gamma=\left\langle x_{1}, x_{2}, x_{3} \mid x_{1}^{2}=x_{2}^{5}=x_{3}^{10}=x_{1} x_{2} x_{3}=1\right\rangle$ onto $G$ : one given by $x_{1} \mapsto(4,6)(5,9)(7,10), \quad x_{2} \mapsto(1,2,3,4,5)(6,7,8,9,10)$ and $x_{3} \mapsto(1,9,8,10,5,6,7,4,3,2)$, and the other given by $x_{1} \mapsto(3,6)(4,8)(5,9)$, $x_{2} \mapsto(1,2,3,4,5)(6,7,8,9,10)$ and $x_{3} \mapsto(1,9,4,6,10,5,8,7,3,2)$. In both cases, conjugates of the image of $x_{2}$ generate the alternating group $A_{10}$, of index 2 in $G$. 
In the first case, let $a$ and $b$ denote the images of $x_{1}$ and $x_{2}$ respectively, and consider the permutation $p=(1,8,9)(2,5,10)(3,7,6)$. Conjugation by $p$ is an automorphism of $A_{10}$ of order 3 , and since also $\left(p(a b)^{-1}\right)^{2}=1$ and $p\left(p(a b)^{-1}\right) p^{-1}=$ $a$, it has the properties required of the element $\alpha$ described in Theorem 5.2(viii). It follows that the action of $G=S_{10}$ in this case can be extended to an action of a semi-direct product $A_{10} \rtimes S_{3}$ on the same surface (where the $S_{3}$ is generated by automorphisms corresponding to conjugation by $p$ and an odd involution in $S_{10}$ which inverts $p$ ).

In the second case, however, there is no such permutation $p$ in $S_{10}$ (an observation which may be checked using MAGMA, or by hand using only the conditions that $p^{3}=1$ and $\left.p^{-1} c^{2} p=b\right)$. Since every automorphism of $A_{10}$ is induced by conjugation by some element of $S_{10}$, it follows that there is no way of extending the action of $G$ in the way prescribed by Theorem 5.2 (viii). Therefore $S_{10}$ is the full automorphism group of the surface in this case.

\section{NON-CYCLIC ABELIAN GROUPS OF AUTOMORPHISMS}

In this section we apply the above results to a special family of groups of automorphisms, namely non-cyclic abelian groups. The extendability of cyclic group actions was studied in [3] and [1].

First we consider the implications of Theorem 5.1 for the non-triangular signatures.

Case 5.1(i): An abelian group $G$ acting with signature $(2 ;-)$ has (partial) monodromy presentation $G=\langle a, b, c, d \mid[a, b][c, d]=\cdots=1\rangle$. Since $G$ is abelian, the relation $[a, b][c, d]=1$ is redundant, and so $G$ can be any finite abelian group generated by four (or fewer) elements. Also the assignment required by Theorem 5.1(i) is $a \mapsto a^{-1}, b \mapsto b^{-1}, c \mapsto c^{-1}, d \mapsto d^{-1}$. Inversion of all elements is an automorphism of any abelian group, and so the action of $G$ is always extendable.

Case 5.1(ii): An abelian group $G$ acting with signature $(1 ; t, t)$ has (partial) monodromy presentation $G=\left\langle a, b, x \mid x^{t}=([a, b] x)^{t}=\cdots=1\right\rangle$. Since $G$ is abelian, the second relation $([a, b] x)^{t}=1$ is redundant, and $G$ can be any finite abelian group generated by three (or fewer) elements, at least one of which has order $t$. The assignment required by Theorem [5.1(ii) is $a \mapsto a^{-1}, b \mapsto b^{-1}, x \mapsto x^{-1}$. This corresponds to an automorphism of $G$, and therefore the action of $G$ is always extendable.

Case 5.1(iii): This case is impossible for an abelian group $G$, since the Fuchsian group presentation for signature $(1 ; t)$ is $\left\langle a, b \mid[a, b]^{t}=1\right\rangle$, and the relation $[a, b]^{t}=$ 1 forces $t=1$ (for a smooth embedding).

Case 5.1(iv): An abelian group $G$ acting with signature $(0 ; t, t, u, u)$ has (partial) monodromy presentation $G=\left\langle a, b, c, d \mid a^{t}=b^{t}=c^{u}=d^{u}=a b c d=\cdots=1\right\rangle$. The relation $a b c d=1$ implies $a b=(c d)^{-1}$, which has order dividing both $t$ (the common orders of $a$ and $b$ ) and $u$ (the common orders of $c$ and $d$ ), since $G$ is abelian. Since also $b=a^{-1}(a b)$ and $d=(a b c)^{-1}$, the group $G$ can be generated by the elements $a, a b$, and $c$, and hence the order of $G$ must divide $t m u$, where $m=\operatorname{gcd}(t, u)$.

In particular, $G$ must be a factor group of $C_{t} \oplus C_{m} \oplus C_{u}$, in which the orders of the generators $a, b, c, d$ are preserved (as $t, t, u, u$ respectively). The assignment required by Theorem [5.1(iv) is $a \mapsto b, b \mapsto a, c \mapsto d, d \mapsto c$, which implies that the element $a b=(c d)^{-1}$ is fixed. 
Clearly this assignment corresponds to an automorphism of $G$ in the case where $G \cong C_{t} \oplus C_{m} \oplus C_{u}$ (that is, where no further relations hold other than those which follow from the prescription of the orders of the generators and the fact that they commute). It will also give an automorphism in other cases, for example when $a=b^{-1}$ and $c=d^{-1}$ and $G=\langle a, c\rangle \cong C_{t} \oplus C_{u}$. The assignment will only fail to give an automorphism in cases where the generators $a, b, c, d$ satisfy additional relations, not all of which continue to hold when $a$ and $c$ are interchanged with $b$ and $d$ respectively.

Whenever this assignment does provide an automorphism of $G$, the action of $G$ is extendable in the manner described in case N5 in Section 3

Similarly, in the special case where $u=t$, if the relations satisfied by the generators $a, b, c, d$ still hold also when $a$ and $b$ are interchanged with $d$ and $c$ respectively, then the action of $G$ is extendable in the manner described in case N4 in Section 3. This is certainly true when $G \cong C_{t} \oplus C_{t} \oplus C_{t}$, and in many other instances besides.

We now consider the implications of Theorem 5.2 for the triangular signatures. In each case the non-cyclic abelian group $G$ is assumed to act with signature $\left(0 ; m_{1}, m_{2}, m_{3}\right)$ and to have a corresponding (partial) monodromy presentation of the form $G=\left\langle a, b, c \mid a^{m_{1}}=b^{m_{2}}=c^{m_{3}}=a b c=\cdots=1\right\rangle$.

Case [5.2(i): Here the signature is $(0 ; t, t, t)$, and the assignment required by Theorem 5.2(i) is $a \mapsto b, b \mapsto c$ and $c \mapsto a$.

Let $A=\langle a\rangle$ and $B=\langle b\rangle$, and suppose $A \cap B$ has order $m$ and hence index $k=t / m$ in each of $A$ and $B$. Then $G=\langle a, b\rangle=A B$, and so $|G|=|A B|=$ $|A||B| /|A \cap B|=t^{2} / m=t k$, and $G \cong C_{t} \oplus C_{k}$. Also $A \cap B$ is cyclic and is generated by each of $a^{k}$ and $b^{k}$; so $b^{k}=\left(a^{k}\right)^{s}$ for some $s$ coprime to $m=t / k$. Now if the assignment $a \mapsto b, b \mapsto c, c \mapsto a$ gives an automorphism of $G$, then the relation $b^{k}=\left(a^{k}\right)^{s}$ must be preserved by this assignment. So $(a b)^{-k}=c^{k}=\left(b^{k}\right)^{s}$, and therefore $1=(a b)^{k}\left(b^{k}\right)^{s}=a^{k} b^{k}\left(b^{k}\right)^{s}=a^{k}\left(a^{k}\right)^{s}\left(\left(a^{k}\right)^{s}\right)^{s}=\left(a^{k}\right)^{1+s+s^{2}}$, which implies that $s^{2}+s+1 \equiv 0(\bmod m)$. Conversely, if $b^{k}=\left(a^{k}\right)^{s}$, where $s^{2}+s+1 \equiv 0$ $(\bmod m)$, then the required assignment gives an automorphism of $G$.

Case 5.2(ii): This case is similar to the one above. The signature is $(0 ; t, t, u)$, and the assignment required by Theorem 5.2 (ii) is $a \mapsto b \mapsto a$ and $c \mapsto c$. Since $G$ is abelian, the order $u$ of $a b$ is a divisor of $t=|\langle a\rangle|=|\langle b\rangle|$. Letting $A=\langle a\rangle$ and $B=\langle b\rangle$, and $m=|A \cap B|$ and $k=t / m$, we have $G=A B \cong C_{t} \oplus C_{k}$, and $b^{k}=\left(a^{k}\right)^{s}$ for some $s$ coprime to $m=t / k$. Note also that $1=(a b)^{u}=a^{u} b^{u}$. So $a^{u}=b^{-u}$, and hence both $a^{u}$ and $b^{u}$ lie in $A \cap B$, so that $k=t / m$ divides $u$. Now if the required assignment $a \mapsto b, b \mapsto a, c \mapsto c$ gives an automorphism of $G$, then $a^{k}=\left(b^{k}\right)^{s}=\left(\left(a^{k}\right)^{s}\right)^{s}$, and therefore $s^{2} \equiv 1(\bmod m)$. Conversely if $b^{k}=\left(a^{k}\right)^{s}$, where $s^{2} \equiv 1(\bmod m)$, then the required assignment gives an automorphism of $G$. Cases 5.2(iii)-(vi): In case 5.2(iii) the signature is $(0 ; 2,7,7)$. This requires the product of the elements $b$ and $c$ of order 7 to have order 2, which is impossible for an abelian group. Analogous arguments exclude signatures $(0 ; 3,3,7),(0 ; 3,8,8)$ and $(0 ; 4,4,5)$.

Cases 5.2(vii)-(viii): In case 5.2(vii) the signature is $(0 ; 3, n, 3 n)$, requiring the product of elements of orders 3 and $n$ to have order $3 n$. For an abelian group $G$, this occurs only when $G$ is cyclic (of order $3 n$ ), as treated in [3], 11]. The analogous argument excludes also the signature $(0 ; 2, n, 2 n)$.

We can now summarise the conditions for the extendability of non-cyclic abelian groups in the following theorem. 
Theorem 7.1. Let $G$ be a non-cyclic finite abelian group acting on a compact Riemann surface $X$ of genus $g$ with non-maximal Fuchsian signature $\sigma$. Then the following hold:

(a) The signature $\sigma$ cannot be one of $(1 ; t),(0 ; 2,7,7),(0 ; 3,3,7),(0 ; 3,8,8)$, $(0 ; 4,4,5),(0 ; 3, n, 3 n)$ or $(0 ; 2, n, 2 n)$.

(b) If $\sigma=(2 ;-)$ or $(1 ; t, t)$, then $G \neq$ Aut $X$.

(c) If $\sigma=(0 ; t, t, u, u)$, then $G$ is a factor group of $C_{t} \oplus C_{u} \oplus C_{m}$, where $m=$ $\operatorname{gcd}(t, u)$, and further, if the action of $G$ corresponds to a (partial) monodromy presentation in terms of commuting generators $a, b, c, d$ subject to relations $a^{t}=$ $b^{t}=c^{u}=d^{u}=1$, then if the assignment $a \mapsto b \mapsto a, c \mapsto d \mapsto c$ is an automorphism of $G$, then $G \neq$ Aut $X$.

(d) If $\sigma=(0 ; t, t, t)$, then $G \cong C_{t} \oplus C_{k}$ with monodromy presentation of the form $G=\left\langle a, b \mid a^{t}=b^{t}=(a b)^{t}=[a, b]=1, b^{k}=a^{k s}\right\rangle$ for some $k$ dividing $t$ and some $s$ coprime to $t / k$, and in this case $G \neq$ Aut $X$ if and only if $s^{2} \equiv 1(\bmod t / k)$ or $s^{2}+s+1 \equiv 0(\bmod t / k)$.

(e) If $\sigma=(0 ; t, t, u)$ with $t \neq u$, then $u$ divides $t$, and $G \cong C_{t} \oplus C_{k}$ with monodromy presentation $G=\left\langle a, b \mid a^{t}=b^{t}=(a b)^{u}=[a, b]=1, b^{k}=a^{k s}\right\rangle$ for some $k$ dividing $u$ and some $s$ coprime to $t / k$, and in this case $G \neq \operatorname{Aut} X$ if and only if $s^{2} \equiv 1(\bmod t / k)$.

Note that further arithmetic conditions will be satisfied in the last two cases of Theorem [7.1. Suppose we write $t=d u$, where $u$ is the order of $a b$ (with $d=1$ and $u=t$ in case (d)), and consider all $r$ for which $(a b)^{k r}=1$. Then on the one hand, since $a b$ has order $u$, we know $(a b)^{k r}=1$ if and only if $r$ is divisible by $u / k$. On the other hand, if $(a b)^{k r}=1$, then $a^{-k r}=b^{k r}=\left(a^{k s}\right)^{r}$. So $a^{(s+1) k r}=1$, and therefore $r(s+1)$ has to be divisible by $t / k$, and conversely. In particular, taking $r=u / k$, we find that $s+1$ is divisible by $t / u$, that is, by $d$. Writing $s+1=p d$, we now have that $(a b)^{k r}=1$ if and only if $r p d$ is divisible by $t / k$, or equivalently, $r p$ is divisible by $u / k$. Thus $s+1=p t / u$, where $p$ is coprime to $u / k$.

Moreover, when $u=t$ we find that both $s$ and $s+1$ are coprime to $t / k$, and hence $t / k$ must be odd in case (d).

We now consider specific examples, which reveal further which extensions are possible for non-cyclic abelian groups acting with triangular signatures.

Example 7.2. Suppose $G$ acts with signature $(0 ; 7,7,7)$. This is case (d) of Theorem 7.1 with $k=t$ and $G \cong C_{7} \oplus C_{7}$ (since 7 is prime). The action of $G$ (on a surface of genus 15) always extends to one of a larger group. In fact, the action extends to one of a group of order 294 containing $C_{7} \oplus C_{7}$ as a normal subgroup of index 6 , with signature $(0 ; 2,3,14)$, as described in case N7 of Section 3. See also Example 7.4 below for the general case of N7.

Extensions of the type T1 in Section 4 are impossible. For suppose the contrary, namely that $G$ extends to a group $G^{\prime}$ acting with signature $(0 ; 2,3,7)$. Then, by the observations made in Section $4, G^{\prime}$ can be generated by elements $x, y, z$ satisfying the relations $x^{2}=y^{3}=z^{7}=x y z=1$, such that $z=c$, and conjugates of the element $w=\left(y x y^{-1} x\right)^{4}$ generate a normal subgroup $K$ of index 168 in $G^{\prime}$ and index 7 in $G$. In particular, $K$ must be cyclic of order 7 and generated by $w$ itself. But $w=\left(y x y^{-1} x\right)^{4}$ is inverted by conjugation by $x$ and centralised by $x y=z^{-1}=c^{-1} \in G$. Therefore $y w y^{-1}=x x y w y^{-1} x x=x w x=w^{-1}$, which implies $w=y^{3} w y^{-3}=\left(\left(w^{-1}\right)^{-1}\right)^{-1}=w^{-1}$, contradicting the conclusion that $w$ has order 7 . 
Example 7.3. Suppose $G$ acts with signature $(0 ; 9,9,9)$. Then two possibilities can occur: either $G=\left\langle a, b \mid a^{9}=b^{9}=(a b)^{9}=[a, b]=1\right\rangle \cong C_{9} \oplus C_{9}$, or $G=\left\langle a, b \mid a^{9}=b^{9}=(a b)^{9}=[a, b]=1, a^{3}=b^{3}\right\rangle \cong C_{9} \oplus C_{3}$. Extensions of type N7 are possible in both cases. In the first case $G$ acts on a surface of genus 28 , and the action extends to one of a group of order 486 containing $C_{9} \oplus C_{9}$ as a normal subgroup of index 6 , with signature $(0 ; 2,3,18)$. In the second case $G$ acts on a surface of genus 10, and the action extends to one of a group of order 162 containing $C_{9} \oplus C_{3}$ as a normal subgroup of index 6 , again with signature $(0 ; 2,3,18)$.

Extensions of the type T6 in Section 4 are impossible, for in this case if the action of $G$ extends to one of a group $G^{\prime}$ with signature $(0 ; 2,3,9)$, then $G$ must have a normal subgroup $K$ such that $G / K$ is an extraspecial group of order 27, contradicting the fact that $G$ is abelian.

Example 7.4. Suppose $G$ acts with signature $(0 ; t, t, t)$, where $t$ is any integer greater than 3 . In this example we consider conditions under which an extension of type N7 in Section 3 will be possible. This is a combination of cases N6 and N8, and so extendability requires both assignments $(a, b, c) \mapsto(b, c, a)$ and $(a, b, c) \mapsto$ $(b, a, c)$ to give automorphisms of $G$.

Accordingly, the parameter $s$ in the relation $b^{k}=\left(a^{k}\right)^{s}$ in part (d) of Theorem 7.1 has to satisfy both $s^{2}+s+1 \equiv 0$ and $s^{2} \equiv 1(\bmod t / k)$. Together these congruences give $s \equiv-1-s^{2} \equiv-2(\bmod t / k)$, and therefore $0 \equiv s^{2}+s+1 \equiv(-2)^{2}-2+1 \equiv 3$ $(\bmod t / k)$, so that $t / k=1$ or 3 . Conversely, if $k=t$, then $G \cong C_{t} \oplus C_{t}$ (with $A \cap B$ trivial), and the extension is easy. If $k=t / 3$, then $G \cong C_{t} \oplus C_{t / 3}$ (with $A \cap B$ cyclic of order 3$)$ and $\operatorname{gcd}(s, 3)=\operatorname{gcd}(s+1,3)=1$. So $s=1$, which satisfies both $s^{2}+s+1 \equiv 0$ and $s^{2} \equiv 1(\bmod 3)$. Hence the action of $G$ extends whenever $k=t$ or $k=t / 3$, to one of a group containing $G$ as a normal subgroup of index 6 , with signature $(0 ; 2,3,2 t)$.

Example 7.5. Suppose $G$ acts with signature $(0 ; 4,8,8)$, or equivalently, signature $(0 ; 8,8,4)$. Then just two possibilities can occur: either $G=\langle a, b| a^{8}=b^{8}=$ $\left.(a b)^{4}=[a, b]=1, a^{4}=b^{4}\right\rangle \cong C_{8} \oplus C_{4}$, or $G=\langle a, b| a^{8}=b^{8}=(a b)^{4}=[a, b]=$ $\left.1, a^{2}=b^{2}\right\rangle \cong C_{8} \oplus C_{2}$. (Note that if $a^{2}=b^{-2}$, then $a b$ has order 2 rather than 4.) An extension of type $\mathrm{N} 8$ is possible in both cases, to groups of order 64 and 32 acting with signature $(0 ; 2,8,8)$ on surfaces of genus 9 and 5 respectively.

As we will see in Example 7.7 below, the action of $C_{8} \oplus C_{2}$ here on a surface of genus 5 extends also to one of a group of order 64 with signature $(0 ; 2,4,8)$. This is an extension of type T9, which is impossible for $C_{8} \oplus C_{4}$.

We now consider the possibility of a greater extension, of type T4. In applying the observations made in Section 4, we replace $(a, b, c)$ by $(c, a, b)$ for consistency of signature, and take $c=(a b)^{-1}$ as required. If the action of $G$ extends to one of a group $G^{\prime}$ acting with signature $(0 ; 2,3,8)$, then $G^{\prime}$ can be generated by elements $x, y, z$ satisfying the relations $x^{2}=y^{3}=z^{8}=x y z=1$, such that $c=z^{2}, a=$ $y z^{-1} y z y^{-1} z y^{-1}=y x y x y x y^{-1}$ and $b=y^{-1} z^{-2} y z y^{-1} z^{2} y=y^{-1} x y x y x y x y^{-1} x y$, and conjugates of the element $w=\left(y x y^{-1} x\right)^{3}=a^{-2} c$ generate a normal subgroup $K$ of index 96 in $G^{\prime}$ and index 8 in $G$. In particular, $|K|=|G| / 8=2$ or 4 . Now $w=\left(y x y^{-1} x\right)^{3}$ is inverted by conjugation by $x$, as is $y^{-1} w y=\left(x y^{-1} x y\right)^{3}$. If $y$ does not centralise $K$, then $K$ must have order 4 and be generated by two commuting involutions, $w$ and $y^{-1} w y$; but if $w$ is an involution, then $1=w^{2}=\left(a^{-2} c\right)^{2}=$ $a^{-4} c^{2}$, so that $|G|=16=4|K|$, a contradiction. Hence $y$ centralises $K$, and so 
$K=\langle w\rangle$ with $w^{x}=w^{-1}$ and $w^{y}=w$, so $w=w^{a}=w^{y x y x y x y^{-1}}=w^{-1}$, which again forces $w$ to have order 2 , and so $|G|=8|K|=16$.

Hence for extendability of a non-cyclic abelian group $G$ of the type T4 we require $G \cong C_{8} \oplus C_{2}$, with $c^{2}=a^{4}$, or equivalently, $a^{2}=b^{2}$. Indeed, in this case the action of $C_{8} \oplus C_{2}$ on a surface of genus 5 extends to an action of a group of order 192, with signature $(0 ; 2,3,8)$.

Example 7.6. Suppose $G$ acts with signature $(0 ; n, 4 n, 4 n)$, where $n$ is any integer greater than 1 . In this example we consider conditions which allow an extension of type T8 in Section 4 . Here $G \cong C_{4 n} \oplus C_{k}$, where $k$ divides $n$.

By the observations made in Section 4 , if the action of $G$ extends to one of a group $G^{\prime}$ acting with signature $(0 ; 2,3,4 n)$, then $G^{\prime}$ can be generated by elements $x, y, z$ satisfying the relations $x^{2}=y^{3}=z^{4 n}=x y z=1$, such that $a=y^{-1} z^{4} y$ and $c=z$, and conjugates of the element $w=z^{4}=c^{4}$ generate a normal subgroup $K$ of index 24 in $G^{\prime}$ and index 4 in $G$.

Now since $G$ is abelian, $c^{-1}=z^{-1}=x y$ commutes with $a c=y^{-1} z^{4} y z=$ $y x y^{-1} x y^{-1} x y^{-1}$, and so it follows that

$$
a=y^{-1} z^{4} y=y x y^{-1} x y^{-1} x y^{-1} x y=x y y x y^{-1} x y^{-1} x y^{-1}=\left(x y^{-1}\right)^{4}=\left(z^{4}\right)^{x}=w^{x} .
$$

Also $y z^{4} y^{-1}=y\left(y^{-1} x\right)^{4} y^{-1}=\left(x y^{-1}\right)^{4}=w^{x}$, and hence $w^{y}=w^{y^{-1}}=w^{x}=a$. It follows that $w$ is centralised by $y^{2}$, but since $y$ has order 3 , this implies $w$ is centralised by $y$, and hence $a=w^{y}=w=z^{4}=c^{4}$.

Thus $G$ is cyclic, generated by $c$, with $a=c^{4}$ and $b=(c a)^{-1}=c^{-5}$. (Moreover, $n$ divides 6 , as shown in [3.) In particular, the extendability described in case T8 does not apply for non-cyclic abelian groups.

Example 7.7. Suppose $G$ acts with signature $(0 ; n, 2 n, 2 n)$, where $n \geq 3$. Here $G \cong C_{2 n} \oplus C_{k}$, where $k$ divides $n$, and conditions for extendability of type N8 are given in case (e) of Theorem 7.1 In this example we consider conditions which allow an extension of type T9 in Section 4 .

By the observations made in Section 4 , if the action of $G$ extends to one of a group $G^{\prime}$ acting with signature $(0 ; 2,4,2 n)$, then $G^{\prime}$ can be generated by elements $x, y, z$ satisfying $x^{2}=y^{4}=z^{2 n}=x y z=1$, such that $a=y z^{2} y^{-1}, b=y^{2} z y^{2}$ and $c=z$, and conjugates of the element $w=z^{2}=c^{2}$ generate a normal subgroup $K$ of index 8 in $G^{\prime}$ and index 2 in $G$. Now $w=z^{2}=\left(y^{-1} x\right)^{2}$, and so $x w x=\left(x y^{-1}\right)^{2}=$ $y w y^{-1}$; but also $y^{-1} w y$ lies in $K$ and hence also in $G$, and therefore commutes with $x y=c^{-1}$. So $y^{-1} w y=x y\left(y^{-1} w y\right) y^{-1} x=x w x=y w y^{-1}$, showing that $y^{2}$ centralises $w$. In particular, $b^{2}=\left(y^{2} z y^{2}\right)^{2}=y^{2} z^{2} y^{2}=y^{2} w y^{2}=w=c^{2}$, and hence $k=2$ (so $n$ is even) and $G \cong C_{2 n} \oplus C_{2}$. Furthermore, since $c=(a b)^{-1}$, we find $b^{2}=c^{2}=(a b)^{-2}=a^{-2} b^{-2}$ and so $a^{2}=b^{-4}$; hence $K=\left\langle a, b^{2}\right\rangle \cong C_{n} \oplus C_{2}$.

We claim that the action of $G$ is always extendable in the manner described in this case. To begin with, the group $G$ has an automorphism $\alpha$ of order 2 that interchanges $b$ with $c$ (and centralises $a$ ). So by case N8 the action of $G$ extends to one of the group generated by $\alpha$ and $c$ with signature $(0 ; 2,2 n, 2 n)$. It is then a simple exercise to verify that this group also has an automorphism $\beta$ that interchanges $\alpha c$ with $c^{-1}$, and hence (again by case N8) the action of $\langle\alpha, c\rangle$ extends to one of the group generated by $\beta$ and $c$, with signature $(0 ; 2,4,2 n)$. The concatenation of these two extensions of type $\mathrm{N} 8$ gives the required extension of type T9, and a group of $16 n$ automorphisms of a surface of genus $2 n-3$ (for every even $n$ ). 


\section{REFERENCES}

[1] A. F. Beardon, The Geometry of Discrete Groups, Graduate Texts in Mathematics, 91, Springer, New York, 1983. MR 85d:22026

[2] W. Bosma, J. Cannon and C. Playoust, The Magma Algebra System I: The User Language, J. Symbolic Comput. 24 (1997), 235-265.

[3] E. Bujalance and M. Conder, On cyclic groups of automorphisms of Riemann surfaces, J. London Math. Soc. (2) 59 (1999), 573-584. MR 2000g:20098

[4] E. Bujalance, J. M. Gamboa and G. Gromadzki, The full automorphism groups of hyperelliptic Riemann surfaces, Manuscripta Math. 79 (1993), 267-282. MR 94f:20093

[5] F. J. Cirre, Automorphism groups of real algebraic curves which are double covers of the real projective plane, Manuscripta Math. 101 (2000), 495-512. MR 2001f:14055

[6] L. Greenberg, Maximal Fuchsian groups, Bull. Amer. Math. Soc. 69 (1963), 569-573. MR 26:6127

[7] L. Greenberg, Maximal groups and signatures, in Discontinuous groups and Riemann surfaces, Ann. of Math. Studies, No. 79, (Princeton, 1974), 207-226. MR 52:740

[8] W. J. Harvey, Cyclic groups of automorphisms of a compact Riemann surface, Quart. J. Math. 17 (1966), 86-97. MR 34:1511

[9] C. Maclachlan, Abelian groups of automorphisms of compact Riemann surfaces, Proc. London Math. Soc. (3) 15 (1965), 699-712. MR 31:3596

[10] D. Singerman, Finitely maximal Fuchsian groups. J. London Math. Soc. (2) 6 (1972), 29-38. MR 48:529

[11] D. Singerman and P. Watson, Non-maximal cyclic group actions on compact Riemann surfaces. Rev. Mat. Complutense (2) 10 (1997), 423-439. MR 99a:20050

[12] P. Turbek, An explicit family of curves with trivial automorphism groups. Proc. Amer. Math. Soc. 122 (1993), 657-664. MR 95a:14034

[13] P. Turbek, Sufficient conditions for a group of automorphisms of a Riemann surface to be its full automorphism group. J. Pure Appl. Algebra 123 (1998), 285-300. MR 99c:14045]

[14] A. Wiman, Über die hyperelliptischen Kurven und diejenigen vom Geschlecht $p=3$, welche eindeutige Transformationen in sich zulassen, Bihang Till. Kungl. Svenska VetenskapsAkademiens Handlingar 21, no. 1, (1895), 23 pp.

Departamento de Matemáticas Fundamentales, Facultad de Ciencias, uned, c/ Senda DEL REY S/N, 28040 MADRID, Spain

E-mail address: eb@mat.uned.es

Departamento de Matemáticas Fundamentales, Facultad de Ciencias, Uned, c/ Senda DEL REY S/N, 28040 MADRID, SPAin

E-mail address: jcirre@mat.uned.es

Department of Mathematics, University of Auckland, Private Bag 92019, Auckland, New ZeALAND

E-mail address: conder@math.auckland.ac.nz 